\title{
Data report: bulk rock compositions of samples from the IODP Expedition 309/312 sample pool, ODP Hole 1256D'
}

\author{
Natsuki Neo, ${ }^{2}$ Shusaku Yamazaki, ${ }^{2}$ and Sumio Miyashita ${ }^{2}$
}

\section{Chapter contents}

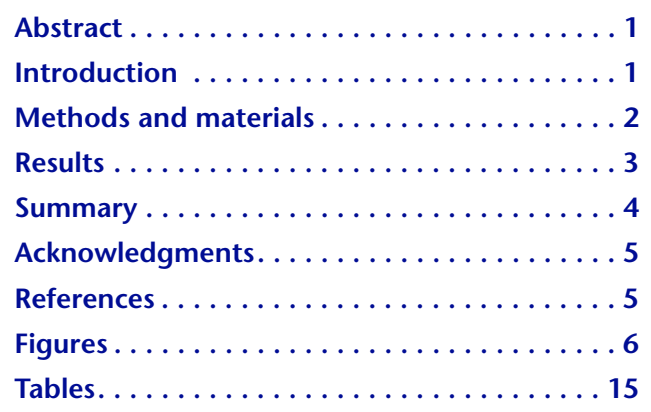

${ }^{1}$ Neo, N., Yamazaki, S., and Miyashita, S., 2009. Data report: bulk rock compositions of samples from the IODP Expedition 309/312 sample pool, ODP Hole 1256D. In Teagle, D.A.H., Alt, J.C., Umino, S., Miyashita, S., Banerjee, N.R., Wilson, D.S., and the Expedition 309/312 Scientists, Proc. IODP, 309/312: Washington, DC (Integrated Ocean Drilling Program Management International, Inc.).

doi:10.2204/iodp.proc.309312.204.2009

${ }^{2}$ Graduate School of Science and Technology, Niigata University, 8050 Ikarashi 2-no-cho, Nishiku, Niigata 950-2181, Japan.

Correspondence author:

f05j007a@mail.cc.niigata-u.ac.jp

\section{Abstract}

In this data report we present major and trace element abundances for 159 samples recovered from Hole 1256D during Integrated Ocean Drilling Program Expedition 309/312, determined by X-ray fluorescence and inductively coupled plasma-mass spectrometry (ICP-MS). These samples represent a large proportion of rocks taken from the Expedition 309/312 sample pool, a collaborative effort to provide comprehensive geochemical analyses of a representative suite of samples from Hole 1256D. The samples analyzed are distributed from the sheet and massive flows ( 750 meters below seafloor [mbsf]), through the sheeted dike complex (1060.9-1406.6 mbsf), and to the late-stage crosscutting dike $(\sim 1500 \mathrm{mbsf})$ at the present bottom of hole underlying the two gabbro intrusions. Analytical procedures and accuracy of analysis are described and compared with shipboard analyses from Expeditions 309 and 312. There are clear compositional differences for some elements between the shipboard analyses and this study. Some of the samples collected during Expedition 312 were not completely dissolved by routine acid digestion, most probably because of the presence of zircon, resulting in low concentrations of elements hosted by zircon including $\mathrm{Zr}, \mathrm{Hf}$, Th, and U. These samples were further analyzed by ICP-MS following alkali fusion.

Most rocks sampled during Expedition 309/312 display normal mid-ocean-ridge basalt (N-MORB) signatures. However, three samples yield trace element patterns with light rare earth element enrichment patterns. The late dike recovered from the present bottom of Hole 1256D has a highly evolved N-MORB chemistry.

\section{Introduction}

Hole $1256 \mathrm{D}$ is in $15 \mathrm{Ma}$ oceanic crust of the Cocos plate, which formed during an episode of superfast ( $220 \mathrm{~mm} / \mathrm{y}$ full rate; Wilson, 1996) spreading of the East Pacific Rise (Fig. F1). Following drilling operations during Ocean Drilling Program (ODP) Leg 206 (Wilson, Teagle, Acton, et al., 2003) and Integrated Ocean Drilling Program (IODP) Expedition 309/312 (see the "Expedition 309/ 312 summary" chapter), Hole 1256D now penetrates 1507.1 meters below seafloor (mbsf) and provides the first complete sampling of intact upper oceanic crust down to gabbros.

Four major lithologic zones were distinguished in Hole 1256D: lavas, transition zone, sheeted dike complex, and plutonic section 
(Fig. F2). The upper part of Hole $1256 \mathrm{D}$ consists of a thick sequence of lavas (lava pond, 250-350.3 mbsf and inflated flows, 350.3-533.9 mbsf) and sheet and massive flows (533.9-1004.2 mbsf) separated by a 60 $\mathrm{m}$ thick transition zone (1004.2-1060.9 mbsf) from the underlying thin sheeted dike complex (1060.91406.6 mbsf) (upper dikes, 1060.9-1348.3 mbsf and granoblastic dikes, 1348.3-1406.6 mbsf). The first gabbroic rocks were encountered at $1406.0 \mathrm{mbsf}$, and these intrusive gabbros extend to $1458.9 \mathrm{mbsf}$. Beneath these gabbros, a $24.2 \mathrm{~m}$ thick dike screen appears between 1458.9 and 1403.1 mbsf, which separates the upper gabbros from a second intrusive gabbro body between 1483.1 and 1495 mbsf. These lower gabbros are underlain by a further dike screen, and the lowermost rocks recovered from Hole 1256D are from a late-stage, crosscutting basaltic dike (1483.1-1507.1 mbsf) (see the "Expedition 309/312 summary" chapter).

The samples analyzed in this study are part of the Expedition 309/312 sample pool. This was a collaborative effort by shipboard scientists to ensure that a representative suite of samples were comprehensively chemically characterized for their major and trace element compositions. All samples were prepared for analysis at the National Oceanography Centre, University of Southampton, United Kingdom. Samples were cleaned by sawing and grinding to remove drilling contamination before multiple ultrasonic washes with millipore (18.2 M 2 ) water to remove contamination. Samples were reduced to a coarse sand grain size using a fly press and reduced to powder by grinding in a Cr-steel shatterbox. Sample powders were then split into 0.5 to $20 \mathrm{~g}$ aliquots and distributed for chemical and isotopic analysis by scientists from the shipboard science party.

The pool samples of Expedition 309/312 provide a representative sample suite from the sheet and massive flows down to the late dike unit of the present bottom of hole. During the IODP Expedition 309/312, major and several trace element abundances were analyzed aboard ship by inductively coupled plasma-atomic emission spectroscopy (ICP-AES) (see the "Expedition 309/312 summary" chapter). In this study, 159 samples from the Expedition 309/312 sample pool were analyzed at Niigata University, Japan, by X-ray fluorescence (XRF) and inductively coupled plasmamass spectrometry (ICP-MS). We report our analytical procedures and our estimates of the precision and accuracy of the analyses. Our new analyses are then compared to the shipboard data, though it should be noted that the pool samples and shipboard samples are not identical and were generally selected from a different positions. Finally, we briefly describe geochemical features of these analyses.

\section{Methods and materials}

Ten major elements ( $\mathrm{Si}, \mathrm{Ti}, \mathrm{Al}, \mathrm{Fe}, \mathrm{Mn}, \mathrm{Mg}, \mathrm{Ca}, \mathrm{Na}, \mathrm{K}$, and P) were analyzed by XRF (Rigaku RIX 3000) at Niigata University following the analytical procedures of Takahashi and Shuto (1997) (Table T1). For trace element analyses (Sc, V, Cr, Co, Ni, Zn, Ga, Rb, $\mathrm{Sr}, \mathrm{Y}, \mathrm{Zr}, \mathrm{Nb}, \mathrm{Cs}, \mathrm{Ba}, \mathrm{La}, \mathrm{Ce}, \mathrm{Pr}, \mathrm{Nd}, \mathrm{Sm}, \mathrm{Eu}, \mathrm{Gd}, \mathrm{Tb}$, $\mathrm{Dy}, \mathrm{Ho}, \mathrm{Er}, \mathrm{Yb}, \mathrm{Lu}, \mathrm{Hf}, \mathrm{Ta}, \mathrm{Pb}, \mathrm{Th}$, and U), 159 samples were dissolved by $\mathrm{HF}^{-\mathrm{HNO}_{3}}$ acid digestion following Takazawa et al. (2003). A total of 62 samples were also dissolved and analyzed by alkali fusion. Solutions were analyzed using an Agilent 7500a ICPMS at Niigata University, calibrated using the reference values for BHVO-1 (basalt; Hawaii, U.S. Geological Survey [USGS]) of Eggins et al. (1997). Although we did not have enough sample powder to analyze all samples, selected trace elements $(\mathrm{V}, \mathrm{Cr}, \mathrm{Ni}, \mathrm{Rb}, \mathrm{Sr}$, $\mathrm{Y}, \mathrm{Zr}, \mathrm{Nb}, \mathrm{Ba}, \mathrm{Pb}$, and $\mathrm{Th}$ ) were also determined by XRF to cross-check the ICP-MS data where possible.

This approach identified that for many samples cored during Expedition 312 and three samples cored during Expedition 309, there had not been complete dissolution during $\mathrm{HF}-\mathrm{HNO}_{3}$ attack, most probably because of the incomplete dissolution of acid-resistant minerals such as zircon and/or titanite. Therefore, all samples from Expedition 312 and three samples from Expedition 309 were dissolved by alkali fusion (Roser et al., 2000). In a variation of the Roser et al. (2000) method we dissolved fluxed samples in concentrated nitric acid as opposed to perchloric acid. Sample powders were dissolved in concentrated $\mathrm{HF}^{-\mathrm{HNO}_{3}}$ using Pt crucibles and evaporated. Afterward, $\mathrm{Na}_{2} \mathrm{CO}_{3}$ alkali flux was added and heated in an electric furnace. The fluxed samples are dissolved in concentrated $\mathrm{HNO}_{3}, \mathrm{HCl}$, and ultrapure water. The samples were finally diluted $\sim 20,000$ times.

To check the accuracy of analyses, GSJ (Geological Survey of Japan) reference samples were analyzed by XRF. Major element data of JB-1a (basalt; Kitamatsuura), JB-2 (basalt; Oshima), and JB-3 (basalt; Fuji) are shown in Table T2. For trace element analyses, USGS Geochemical Reference Standard W-2 (diabase; Virginia) was dissolved and analyzed with every group of samples (Table T3). Major element and trace element abundances of shipboard standards BAS-206 and BAS-312 were also analyzed (Table T3).

For major elements, relative deviation (RD) values, an estimate of accuracy, between this study and recommended values are very low (Table T2). For trace elements, relative standard deviation (RSD) values, an estimate of precision, are $<5 \%$ except for Sc, Co, $\mathrm{Ni}, \mathrm{Pb}$, and $\mathrm{U}$. Trace element $\mathrm{RD}$ values of the aver- 
age results from this study are less than $\pm 4 \%$ for five sets of W-2 analyses compared to the recommended values of Eggins et al. (1997). RSD values of Zr, Hf, $\mathrm{Th}$, and $\mathrm{U}$ that were dissolved by alkali fusion are $<5 \%$, but $\mathrm{RD}$ values for the recommended value (Eggins et al., 1997) are slightly higher (10\%) for eight sets of sample analyses.

Table T3 shows average (five analyses each) values of BAS-206 and BAS-312, RSD values and average standard deviation values for this study. RSD for most elements are $<5 \%$ except for $\mathrm{V}, \mathrm{Cr}, \mathrm{Ni}, \mathrm{Cs}, \mathrm{Ba}$, and $\mathrm{Pb}$.

Sc analyses were strongly affected by a high blank of up to $8 \%$ of BHVO-1 counts per second, and therefore RSD values of Sc were high. Pb blanks were also high, possibly because of contamination by $\mathrm{Pb}$ from plastic solution storage vessels. The contamination of $\mathrm{Pb}$ from plastic vessels is demonstrated by the correlation between residence time in the plastic vessels and measured $\mathrm{Pb}$ abundances in the $5 \% \mathrm{HNO}_{3}$ solutions. Hence, $\mathrm{Pb}$ analyses also show highly dispersed results.

\section{Results}

\section{Comparison of analyses by different dissolution procedures}

Most of the elements analyzed by both dissolution methods (acid digestion and alkali fusion for ICP-MS analyses) show similar values within analytical error ranges. However, $\mathrm{Zr}, \mathrm{Hf}$, Th, and $\mathrm{U}$ concentrations in samples recovered during Expedition 312 show systematic differences between the two dissolution methods. Figure F3 shows a comparison of analyses of the same sample (312-1256D-184R-1, 0-8 cm) dissolved by acid digestion and following alkali fusion. It is apparent that $\mathrm{Zr}, \mathrm{Hf}, \mathrm{Th}$, and $\mathrm{U}$ concentrations are higher in the sample split prepared by alkali fusion compared to that treated by acid digestion. This relationship is true for all samples from Expedition 312 treated by both methods. The discrepancy between the two dissolution methods is interpreted by the presence of acid-resistant minerals containing $\mathrm{Zr}$, $\mathrm{Hf}$, Th, and $\mathrm{U}$, and is discussed below. On the other hand, $\mathrm{Zn}$, Cs, and $\mathrm{Pb}$ show slightly different values between two dissolution methods. As mentioned above, procedural blanks for $\mathrm{Pb}$ are high even for the acid digestion method. The Pb blanks are further increased when samples are prepared by alkali fusion. $\mathrm{Pb}$ abundances in Hole $1256 \mathrm{D}$ samples are significantly affected by high blank levels, but as the $\mathrm{Pb}$ data from acid digestion appear to be more accurate with lower blank levels, these values are used for all samples in this study.
The most remarkable differences in $\mathrm{Zr}$ concentrations yielded by the two dissolution methods is encountered in samples from deeper than 1300 mbsf. The $\mathrm{Zr}$ values by acid digestion are much lower than the $\mathrm{Zr}$ values by alkali fusion for the samples from a deeper level. It is also apparent that the $\mathrm{Zr}$ abundances by acid digestion are much lower than those returned during shipboard analyses of samples from deeper levels than 1300 mbsf (Fig. F4). The Zr abundances by alkali fusion yield concentrations consistent with the shipboard analyses. Furthermore, they show good correlation with XRF data $\left(R^{2}=0.9802\right)$ (Fig. F5). This indicates that the $\mathrm{Zr}$ abundances by acid digestion are incorrect because of the incomplete dissolution of acid-resistant minerals. Consequently, the $\mathrm{Zr}$ values by alkali fusion are regarded as correct values.

Because acid-resistant minerals such as zircon and titanite are not completely dissolved by acid digestion, the elements contained within these minerals will not be correctly analyzed in samples containing these minerals. For example, zircon contains high concentrations of $\mathrm{Zr}$ and $\mathrm{Hf}$, so abundances of $\mathrm{Zr}$ and $\mathrm{Hf}$ are poorly analyzed by acid digestion for the samples containing zircon. Because $\mathrm{Th}$ and $\mathrm{U}$ are also highly compatible with zircon (Mahood and Hildreth, 1983), these concentrations may also be lower than the true values for zircon-bearing samples. Analyses of samples from deeper than 1300 mbsf, prepared by acid digestion only, show discernibly lower $\mathrm{Th}$ and $\mathrm{U}$ concentrations than the analyses by alkali fusion (Fig. F4). We conclude that the presence of zircon in samples deeper than $1300 \mathrm{mbsf}$ has affected our ICP analyses of splits prepared by acid digestion.

The presence of titanite, another common acid-resistant mineral, may result in low abundances of heavy rare earth element (HREE) concentrations in samples prepared by acid digestion due to very high compatibility of HREE into titanite. However, rare earth element (REE) concentrations by the two dissolution methods are in good agreement with each other, suggesting that the presence of titanites has not affected the REE abundances in this study.

In conclusion, we found that three samples (3091256D-146R-1, 30-54 cm; 146R-2, 80-88 cm; and $155 \mathrm{R}-2,60-80 \mathrm{~cm})$ at above $1300 \mathrm{mbsf}$ contain acidresistant minerals because of much lower values for splits prepared by acid digestion than the splits prepared by alkali fusion. Therefore, the solutions dissolved by alkali fusion were used for these three samples to determine $\mathrm{Zr}$, Hf, Th, and $\mathrm{U}$ concentrations.

Therefore, we use the $\mathrm{Zr}$, Hf, Th, and $\mathrm{U}$ analyses which were dissolved by alkali fusion for the samples 
containing acid-resistant minerals. However, because the accuracy of analyses using acid digestion is generally higher than those of alkali fusion, for most trace elements we use data yielded by the standard acid digestion approach. Our preferred trace element analyses are shown in Table $\mathbf{T 4}$ and variations of trace element abundances are shown in Figure F7.

Zircons are usually contained in felsic rocks as accessory minerals. The analyses described above suggest that zircons may be contained in the dike rocks deeper than 1300 mbsf, though zircons were not reported on board (see the "Expedition 309/312 summary" chapter). This may be explained by the different degrees of differentiation in the dike complex, but a systematic difference in Mg-number, where $\mathrm{Mg \#}=100 \times \mathrm{Mg} /(\mathrm{Mg}+\mathrm{Fe})$, is not recognized in samples above 1300 mbsf (Fig. F6). Higher degrees and different styles of hydrothermal alteration may be responsible for the appearance of zircon at the deeper level.

\section{Comparison of data from this study and shipboard data}

During Expedition 309/312, 10 major elements and 11 trace elements (Co, Zn, Sc, Cr, V, Cu, Zr, Y, Sr, Ba, and $\mathrm{Ni}$ ) were analyzed by ICP-AES. Figures $\mathrm{F} 6$ and F7 compare the variations in major element and trace element abundances with depth using both the shipboard data and this study. For the major elements, downhole variations are generally consistent between the shipboard data and this study.

For the trace element analyses, comparison with the shipboard data is possible for the 10 trace elements analyzed on board; however, $\mathrm{Cu}, \mathrm{V}, \mathrm{Cr}, \mathrm{Ni}, \mathrm{Zn}, \mathrm{Y}$, and $\mathrm{Zr}$ values from this study are generally similar to the shipboard data. Sc values are significantly different between shipboard data and this study with Sc concentrations determined during Expedition 309 being much lower than our data. Co abundances determined aboard ship for samples in the vicinity of 1200 mbsf are much higher than our results. Sr abundances from Expedition 312 are in good agreement with our results, but Expedition 309 shipboard data tend to have higher $\mathrm{Sr}$ concentrations than those of this study. Shipboard Ba abundances from 800 to 900 mbsf tend to be higher, and those from 1100 to 1200 mbsf are lower than our results. Some of these discrepancies may be due to the different samples analyzed on board and in this study, but a significant component must be due to differences in analytical methods and analytical equipment and the difficulties of shipboard analysis. Table T5 shows results of BAS-206 and BAS-312 by shipboard and this study for major and trace elements. Major elements by shipboard and this study show generally similar values. However, our data tend to have higher values for some trace elements.

\section{Brief summary of bulk rock chemistries of the basaltic rocks of Hole 1256D}

Almost all basaltic lavas from Expedition 309/312 show normal mid-ocean-ridge basalt (N-MORB) signatures with flat, light rare earth element (LREE)-depleted REE patterns (Fig. F8). Only one analysis (Sample 309-1256D-80R-2, 92-102 cm; $781.47 \mathrm{mbsf}$ ) from the lava succession of Hole 1256D exhibits an LREE-enriched pattern similar to an enriched MORB pattern. Most analyses of the sheeted dike complex including granoblastic dikes also yield N-MORB-like REE patterns. However, two analyses of samples from the same core (Samples 312-1256D-189R-1, 0-13 cm; and 189R-1, 71-89 $\mathrm{cm}$ ) return LREE-enriched patterns (Fig. F8). The late-stage dike (Sample 3121256D-234R-1, 26-29 cm) recovered in the bottommost core from Hole 1256D shows N-MORB patterns but is characterized by highly evolved features (e.g., $\mathrm{Mg} \#=39 ; \mathrm{TiO}_{2}=2.2 \mathrm{wt} \%$ ). This analysis is one of the most evolved analyses throughout Hole 1256D. It is noticed that the uppermost lava pond, suspected to have solidified some $5-10 \mathrm{~km}$ off-axis, also shows relatively evolved chemistry (see the "Expedition $309 / 312$ summary" chapter). This may imply that late-stage magmatism at Site 1256, as displayed by the late dike of the bottom of hole and lava pond of the top of lava sequence, is characterized by evolved magmas.

\section{Summary}

Major and trace element concentrations of 159 pool samples from Expedition 309/312 were analyzed by XRF and ICP-MS. Accuracy of major element analyses is high. Almost all trace elements have values consistent with recommended values. Major element concentrations determined by this study are generally similar to shipboard data as shown with respect to downhole variations. Trace element abundances of $\mathrm{Zr}, \mathrm{Hf}, \mathrm{Th}$, and $\mathrm{U}$ of Expedition 312 samples were analyzed by alkali fusion method because of the presence of zircon.

When compared to shipboard data, our results for trace elements, V, Cr, Ni, Zn, Y, and $\mathrm{Zr}$ are similar in terms of downhole variations. Other elements tend to show wholly or partially different values, may be due to differences in analytical method or differences of analyzed samples.

Almost all basaltic rocks of Expedition 309/312 show N-MORB REE patterns. However, three samples (3091256D-80R-2, 92-102 cm; 312-1256D-189R-1, 0-13 
$\mathrm{cm}$; and 189R-1, 71-89 cm) show LREE-enriched patterns. Moreover, one sample (312-1256D-234R-1, 26-29 $\mathrm{cm}$ ) from the bottom of hole has highly evolved N-MORB chemistry. It may imply that latestage magmatism as shown by the late dike and lava pond that formed the uppermost basement at Site 1256, is characterized by evolved magmas.

\section{Acknowledgments}

We thank the IODP Expedition 309/312 Science Party, the crew, and technical staff of the R/V JOIDES Resolution for their help. This project used samples and/or data provided by the Integrated Ocean Drilling Program (IODP). We thank Damon Teagle and his dedicated team of "rock-crushers" at the National Oceanography Centre, University of Southampton, for the preparation and distribution of Expedition 309/312 sample pool powders. Natsuki Neo, Shusaku Yamazaki, and Sumio Miyashita were supported by the Center of Deep Earth Exploration (CDEX) for travel fares. This study was supported by Grant in Aid of Ministry of Education and Science of Japan (17340162) and Grant for Promotion of Niigata University Project for Sumio Miyashita. We thank Yoshiko Adachi for discussions. This paper is greatly improved by critical review by Damon Teagle. We also thank for reviews by Susumu Umino, John MacLennan and Naoto Hirano, which improved substantially this paper.

\section{References}

Eggins, S.M., Woodhead, J.D., Kinsley, L.P.J., Mortimer, G.E., Sylvester, P., McCulloch, M.T., Hergt, J.M., and Handler, M.R., 1997. A simple method for the precise determination of $\geq 40$ trace elements in geological samples by ICPMS using enriched isotope internal standardisation. Chem. Geol., 134(4):311-326. doi:10.1016/ S0009-2541(96)00100-3

Expedition 309/312 Scientists, 2006. Expedition 309/312 summary. In Teagle, D.A.H., Alt, J.C., Umino, S., Miyashita, S., Banerjee, N.R., Wilson, D.S., and the Expedition 309/312 Scientists. Proc. IODP, 309/312:
Washington, DC (Integrated Ocean Drilling Program Management International, Inc.). doi:10.2204/ iodp.proc.309312.101.2006

Imai, N., Terashima, S., Itoh, S., and Ando, A., 1995. 1994 compilation of analytical data for minor and trace elements in seventeen GSJ geochemical reference samples, "igneous rock series." Geostand. Geoanal. Res., 19(2):135-213. doi:10.1111/j.1751908X.1995.tb00158.x

Mahood, G., and Hildreth, W., 1983. Large partition coefficients for trace elements in high-silica rhyolites. Geochim. Cosmochim. Acta, 47(1):11-30. doi:10.1016/00167037(83)90087-X

Roser, B., Kimura, J., and Hisatomi, K., 2000. Whole-rock elemental abundances in sandstones and mudrocks from the Tanabe Group, Kii Peninsula, Japan. Shimane Diagaku Chikyu Shigen Kankyogaku Kenkyu Hokoku, 19:101-112.

Sun, S.-S., and McDonough, W.F., 1989. Chemical and isotopic systematics of oceanic basalts: implications for mantle composition and processes. In Saunders, A.D., and Norry, M.J. (Eds.), Magmatism in the Ocean Basins. Geol. Soc. Spec. Publ., 42:313-345.

Takahashi, T., and Shuto, K., 1997. Major and trace element analyses of silicate rocks using X-ray fluorescence spectrometry RIX3000. Rigaku-Denki J., 28:25-37. (in Japanese)

Takazawa, E., Okayasu, T., and Satoh, K., 2003. Geochemistry and origin of the basal lherzolites from the northern Oman ophiolite (northern Fizh block). Geochem., Geophys., Geosyst., 4(2):8605. doi:10.1029/2001GC000232

Terashima, S., Taniguchi, M., Mikoshiba, M., and Imai, N., 1998. Preparation of two new GSJ geochemical reference materials: basalt JB-1b and coal fly ash JCFA-1. Geostand. Geoanal. Res., 22(1):113-117. doi:10.1111/ j.1751-908X.1998.tb00550.x

Wilson, D.S., 1996. Fastest known spreading on the Miocene Cocos-Pacific plate boundary. Geophys. Res. Lett., 23(21):3003-3006. doi:10.1029/96GL02893

Wilson, D.S., Teagle, D.A.H., Acton, G.D., et al., 2003. Proc. ODP, Init. Repts., 206: College Station, TX (Ocean Drilling Program). doi:10.2973/odp.proc.ir.206.2003

Initial receipt: 14 October 2008

Acceptance: 3 March 2009

Publication: 17 June 2009

MS 309312-204 
Figure F1. Seafloor topography of the East Pacific Rise and eastern equatorial Pacific Ocean. Hole 1256D is on 15 Ma oceanic crust of the Cocos plate.

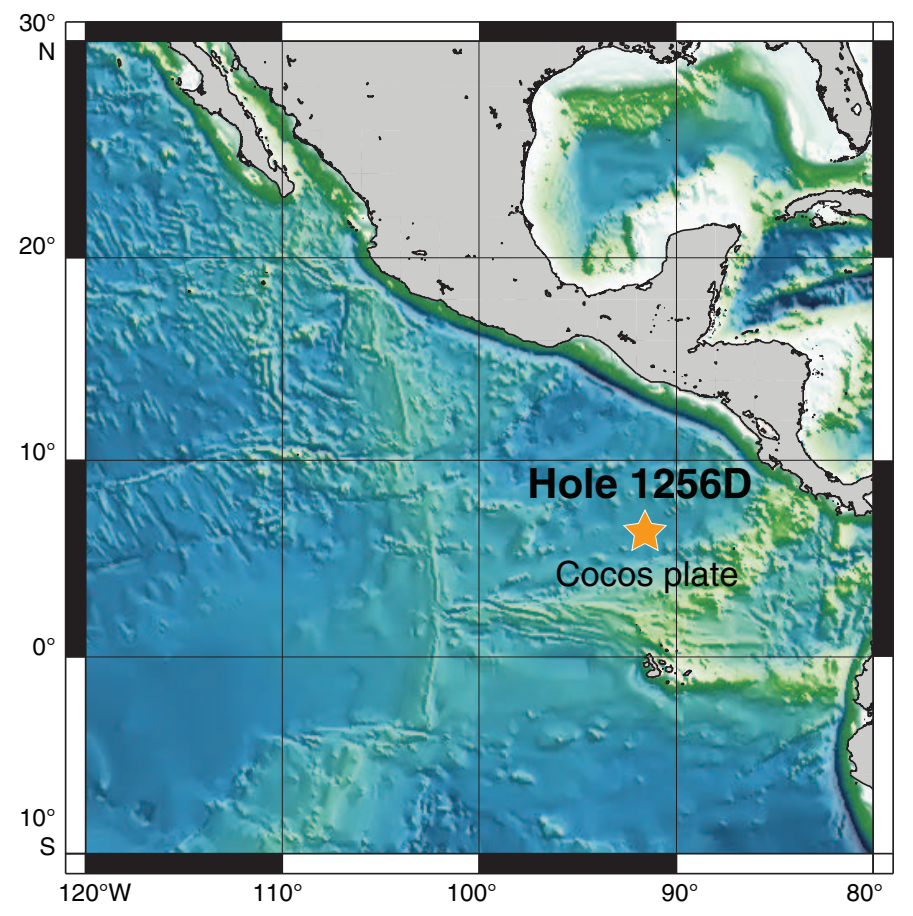


Figure F2. Simplified igneous stratigraphy of Hole 1256D following drilling operations on ODP Leg 206 and IODP Expedition 309/312, modified from Teagle et al. (2006).

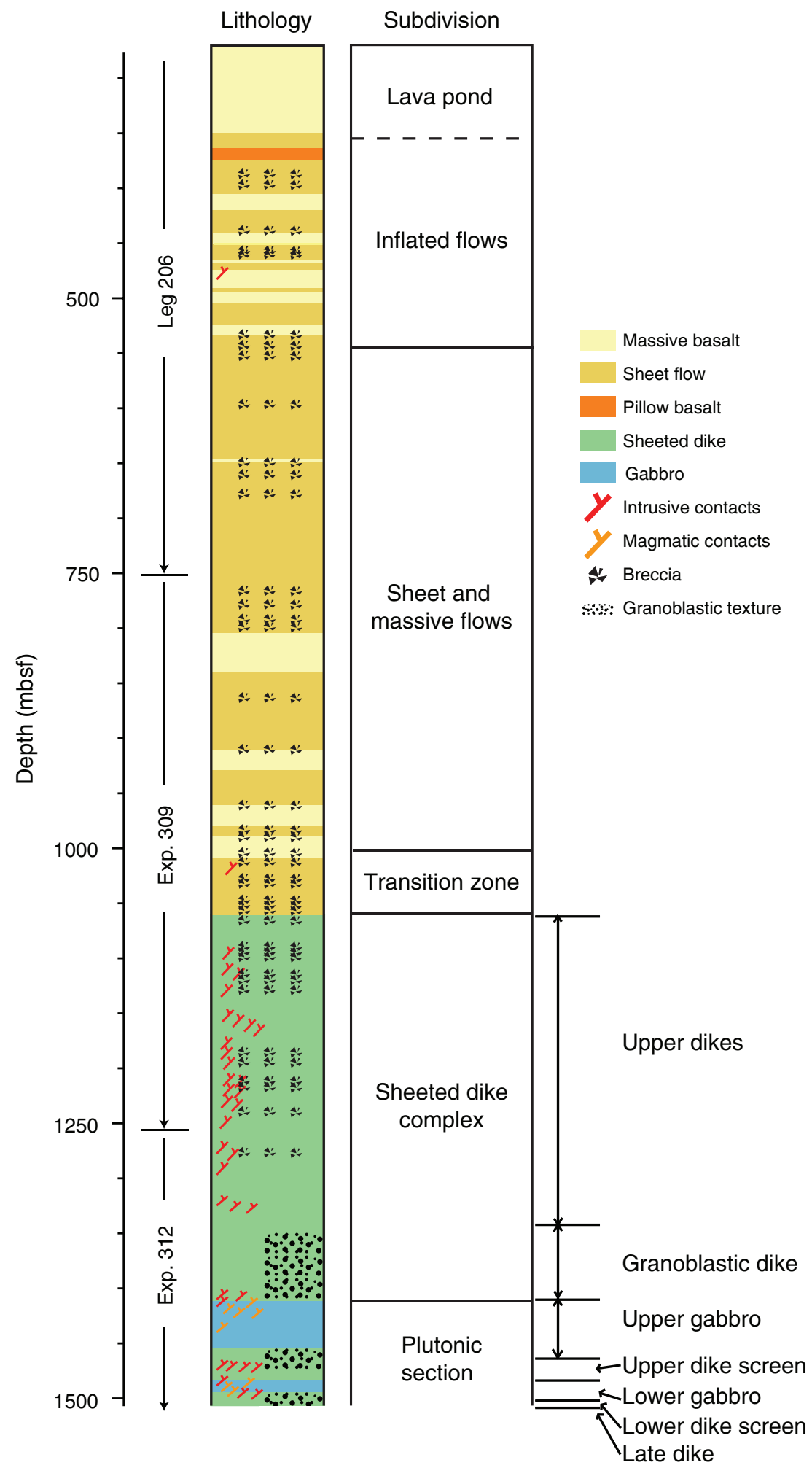


Figure F3. Comparison of inductively coupled plasma-mass spectrometry analyses of Sample 312-1256D-184R$1,0-8 \mathrm{~cm}$, dissolved by acid digestion and following alkali fusion. $\mathrm{Zr}, \mathrm{Hf}$, Th, and $\mathrm{U}$ values by acid digestion are lower than those by alkali fusion, indicating the presence of acid-resistant minerals.

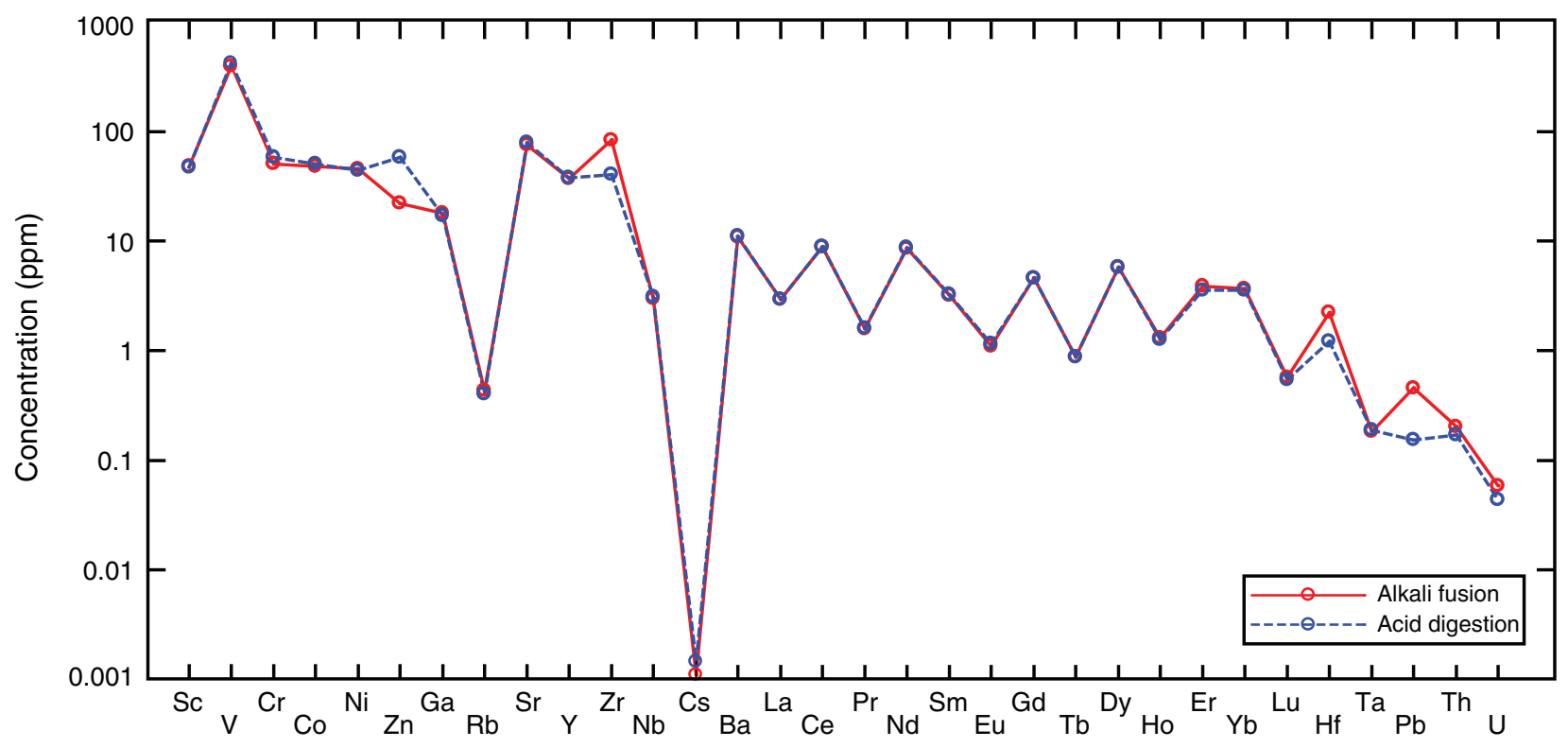


Figure F4. Downhole variations of $\mathrm{Zr}$, Hf, Th, and U abundances of inductively coupled plasma-mass spectrometry analyses by acid digestion and alkali fusion. The discrepancy between the two dissolution methods becomes prominent in samples from deeper than 1300 mbsf.

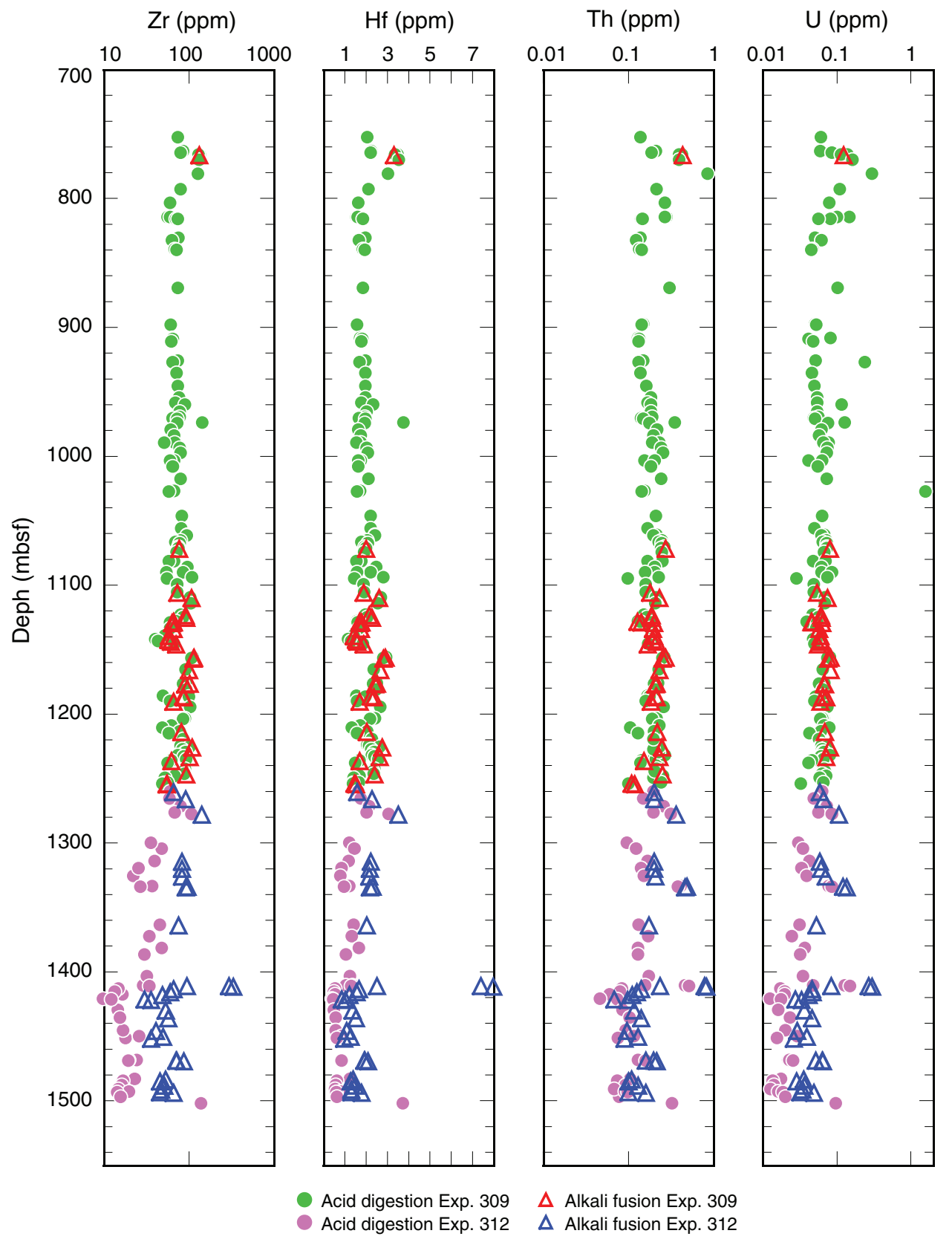


Figure F5. Plot of Zr values of inductively coupled plasma-mass spectrometry (ICP-MS) analyses by alkali fusion method vs. analyses of X-ray fluorescence (XRF) for the same samples. Zr values of ICP-MS analyses show higher concentrations than those of XRF analyses.

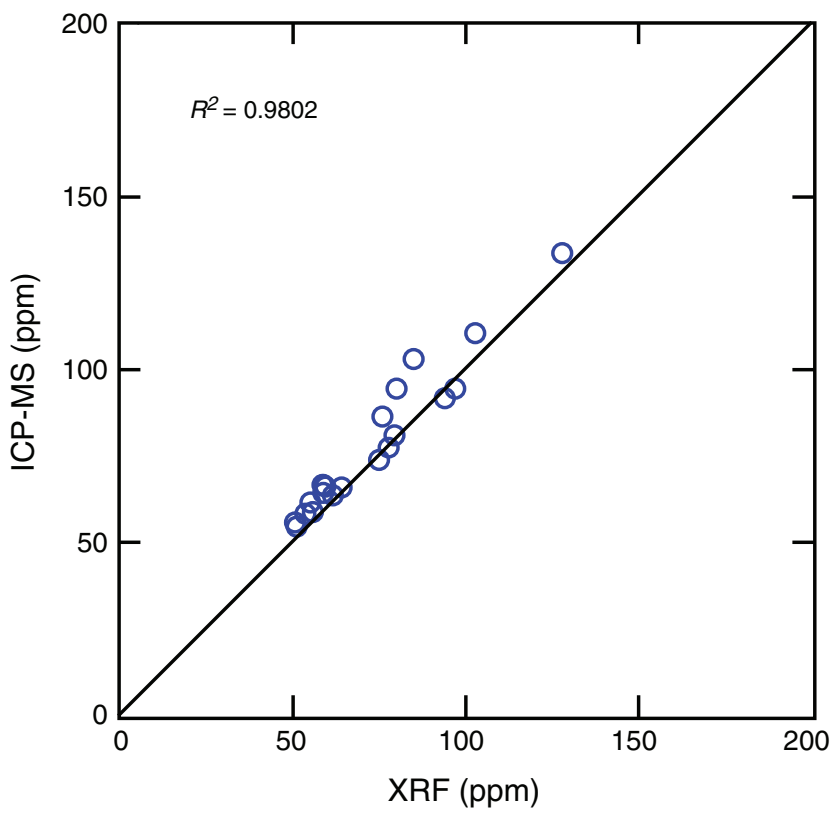


Figure F6. Downhole variation diagram of major elements of the pool sample contents by X-ray fluorescence analyses. Major element concentrations are recalculated on an anhydrous basis and total $\mathrm{Fe}$ as $\mathrm{FeO}$. Blue bands = gabbro intervals. $\mathrm{LOI}=$ loss on ignition. Magnesium number $(\mathrm{Mg} \#)$ $=100 \times \mathrm{Mg} /(\mathrm{Mg}+\mathrm{Fe})$.

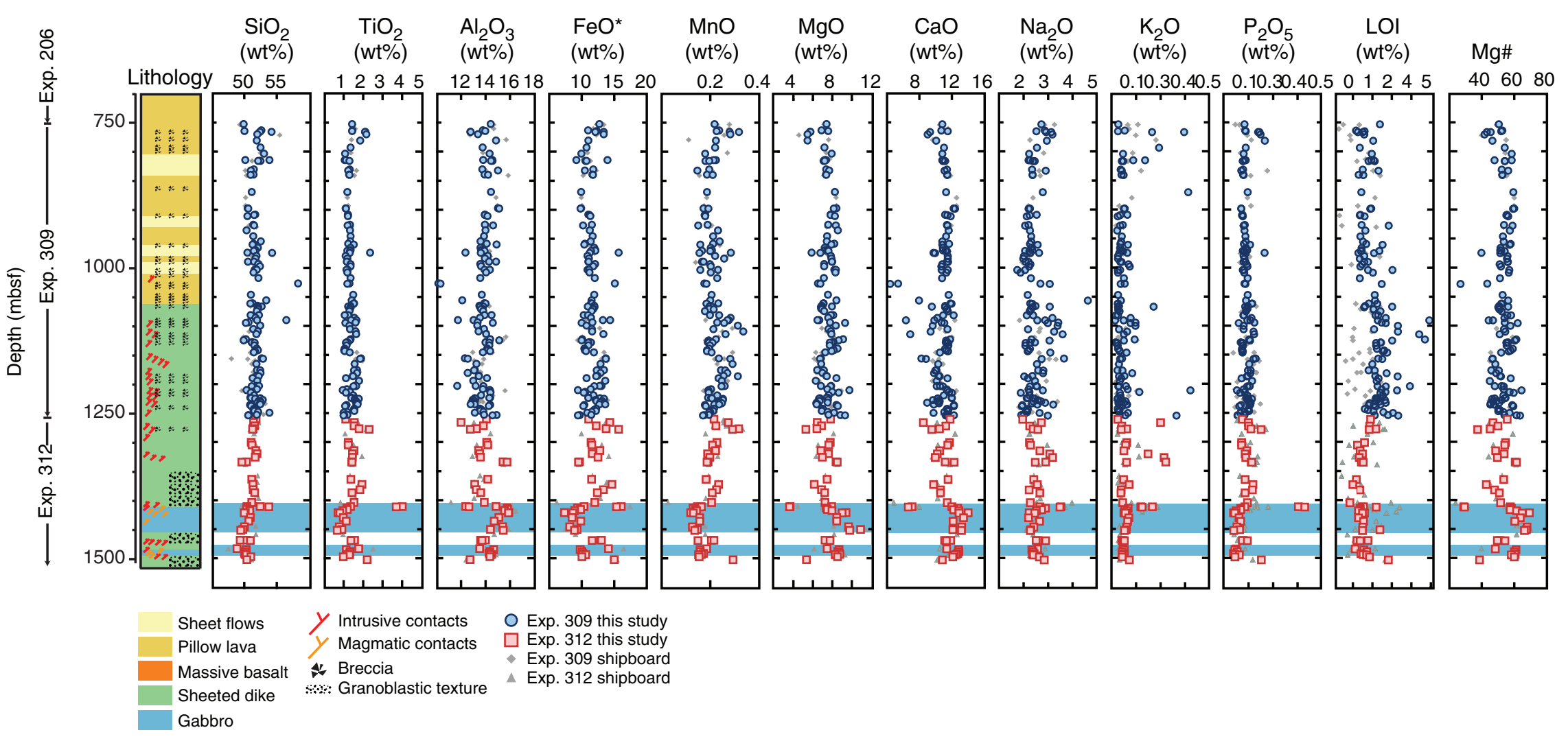


Figure F7. Downhole variation diagram of trace element abundances of the pool samples by inductively coupled plasma-mass spectrometry analyses of this study. Shipboard analyses data of $\mathrm{Co}, \mathrm{Zn}, \mathrm{Sc}, \mathrm{Cr}, \mathrm{V}, \mathrm{Zr}, \mathrm{Y}, \mathrm{Sr}, \mathrm{Ba}$, and Ni abundances by inductively coupled plasma-atomic emission spectroscopy from Expedition 309/312 are also plotted. Blue bands = gabbro intervals. (Continued on next page.)

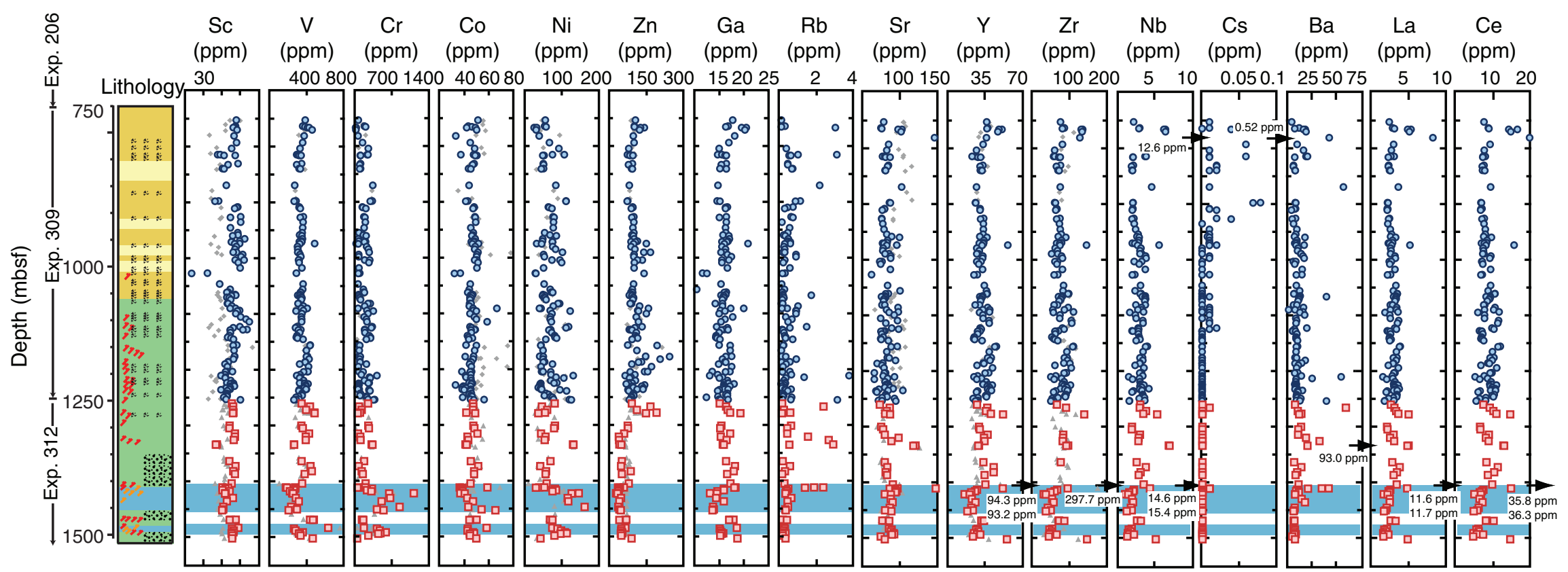

$$
\begin{array}{lll}
\text { Sheet flows } & \text { y Intrusive contacts } & \text { O Exp. } 309 \text { this study } \\
\text { Pillow lava } & y \text { Magmatic contacts } & \square \text { Exp. } 312 \text { this study } \\
\text { Massive basalt } & \text { Execcia } & \text { Exp. } 309 \text { shipboard }
\end{array}
$$

$\triangle$ Exp. 312 shipboard

Gabbro 
Figure F7 (continued).

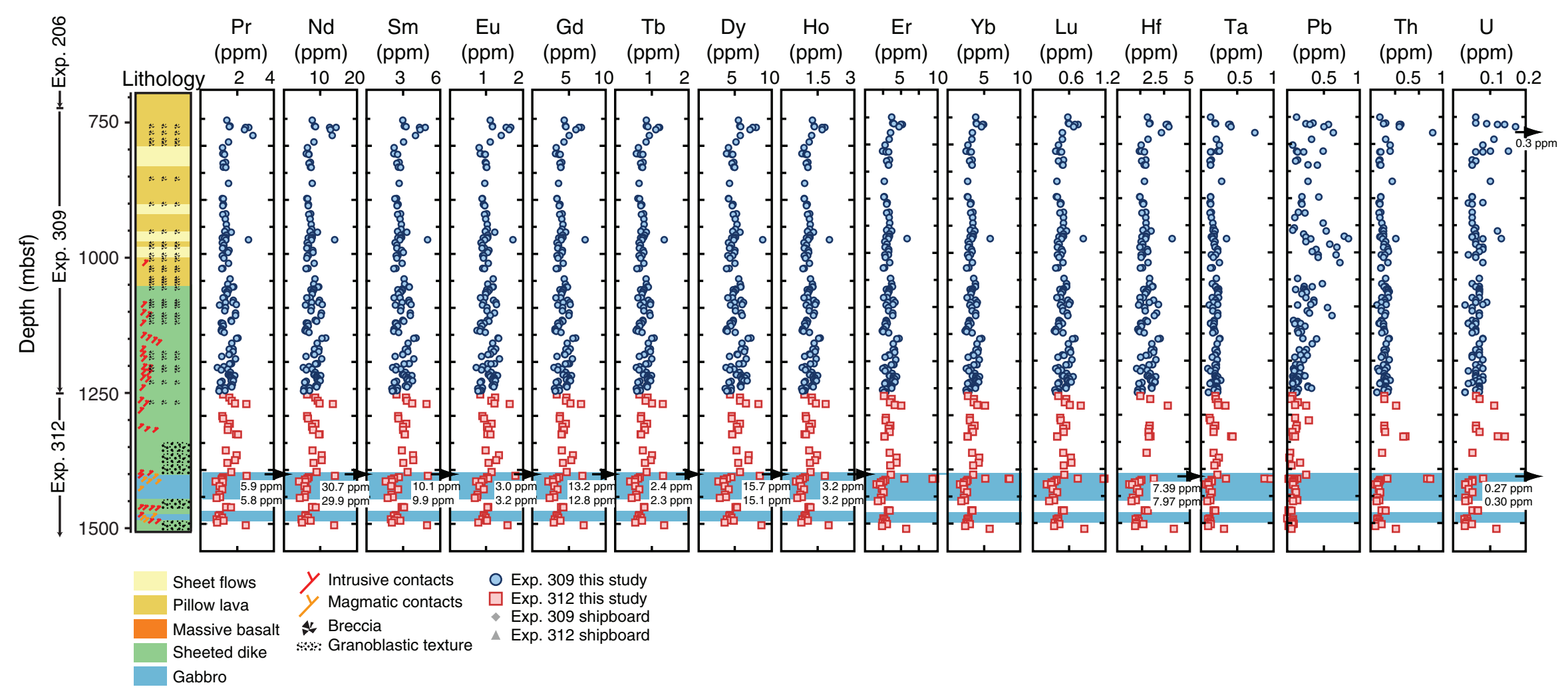


Figure F8. Chondrite (Sun and McDonough, 1989) normalized rare earth element (REE) abundances of the pool samples recovered from Expedition 309/312. REE patterns are shown for each sublithology. Almost all samples show normal mid-ocean-ridge basalt (N-MORB) REE patterns. However, one lava sample from the sheeted and massive flows and two samples from upper dikes show light rare earth element-enriched patterns. A late dike sample from the bottom of Hole 1256D has evolved values. Red lines $=$ N-MORB pattern (Sun and McDonough, 1989), gray lines $=$ REE abundances from $0^{\circ}-10^{\circ} \mathrm{N}$ on the East Pacific Rise (PetDB).
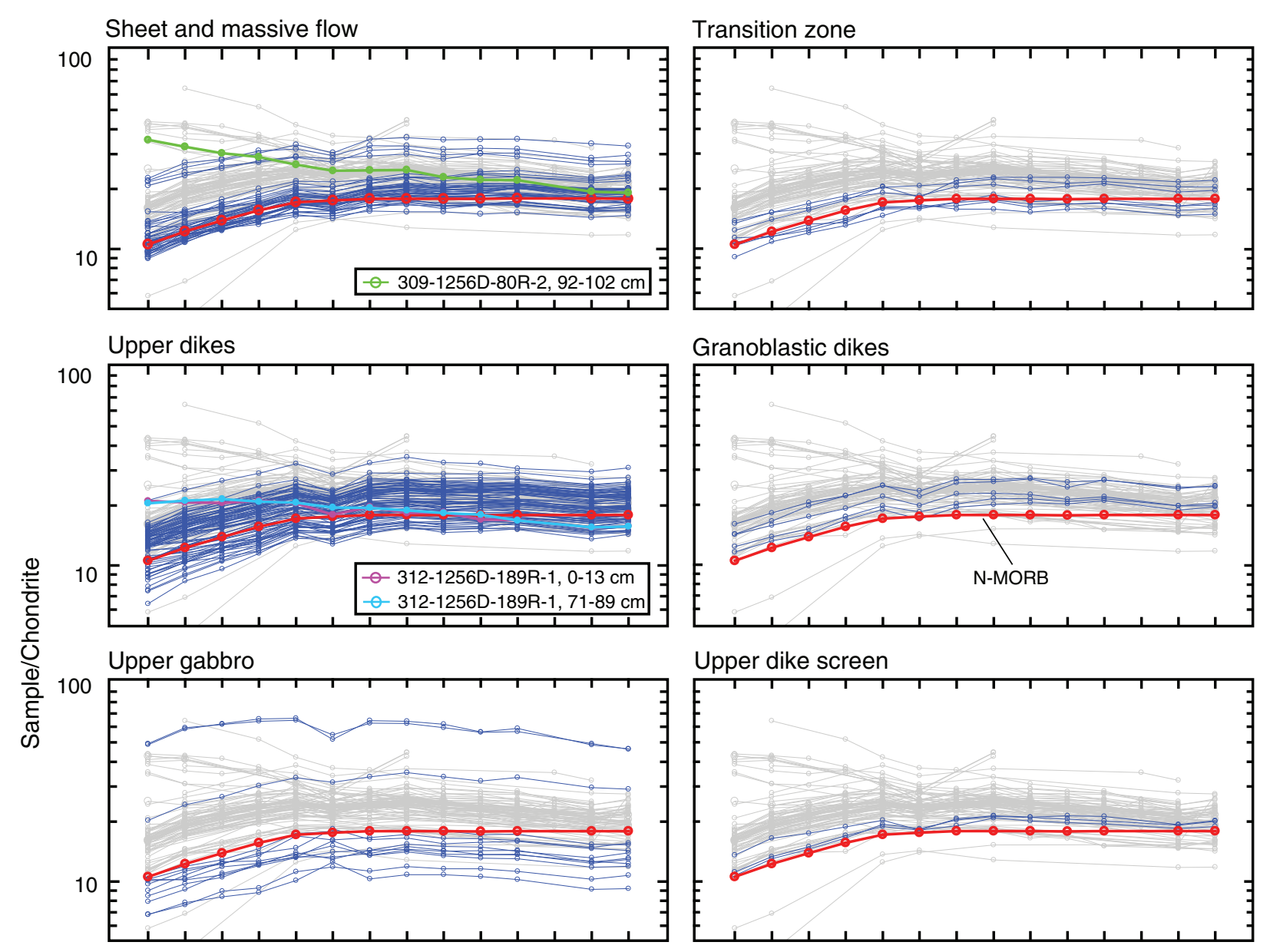

Granoblastic dikes
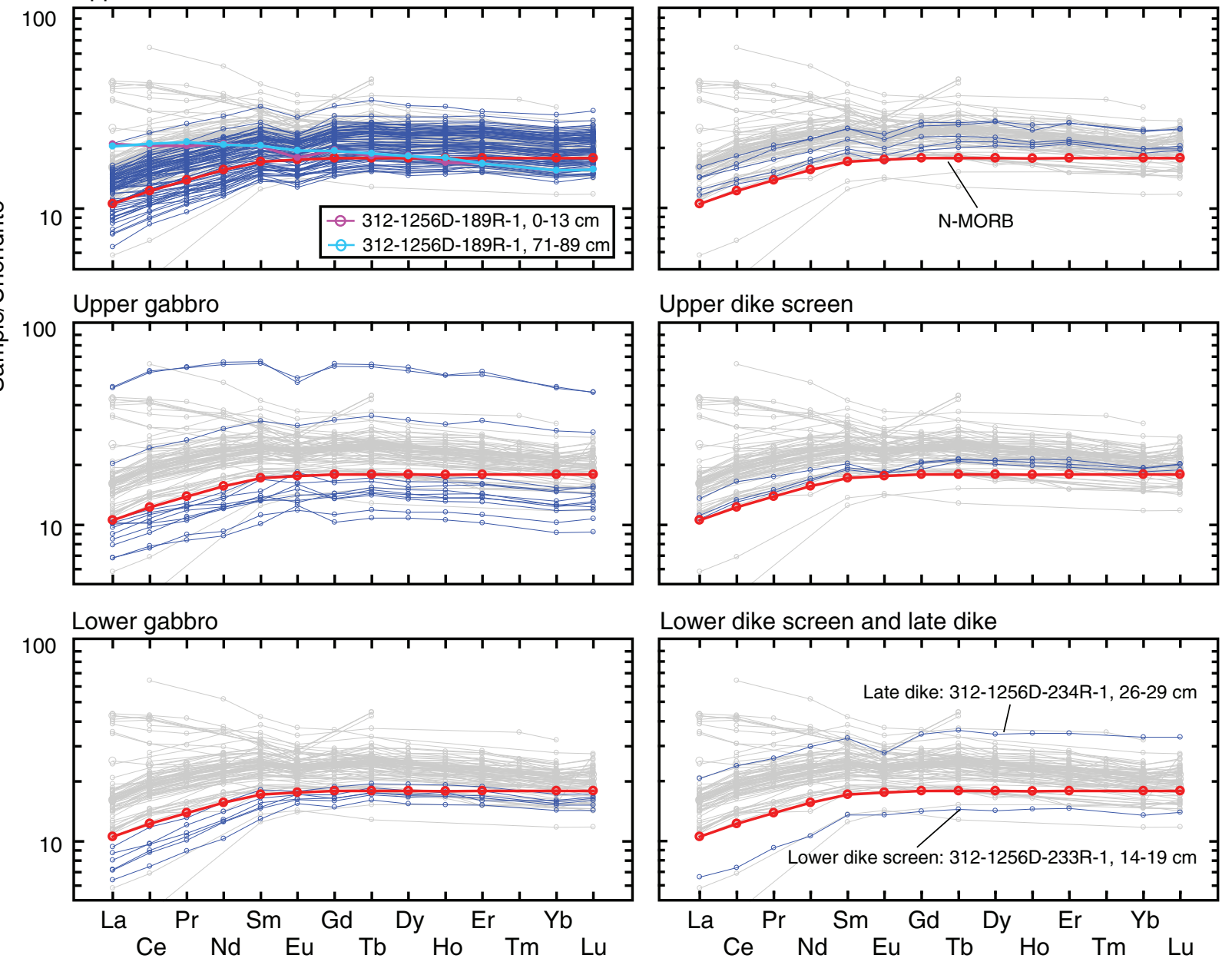

Lower dike screen and late dike

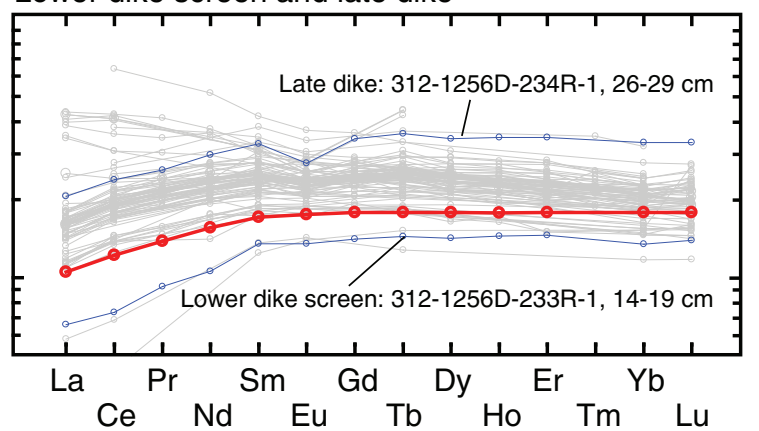


Table T1. Major element abundances. (See table note.) (Continued on next two pages.)

\begin{tabular}{|c|c|c|c|c|c|c|c|c|c|c|c|c|c|c|}
\hline $\begin{array}{l}\text { Core, section, } \\
\text { interval }(\mathrm{cm})\end{array}$ & $\begin{array}{l}\text { Depth } \\
\text { (mbsf) }\end{array}$ & $\begin{array}{l}\mathrm{SiO}_{2} \\
\text { (wt\%) }\end{array}$ & $\begin{array}{l}\mathrm{TiO}_{2} \\
\text { (wt\%) }\end{array}$ & $\begin{array}{l}\mathrm{Al}_{2} \mathrm{O}_{3} \\
\text { (wt\%) }\end{array}$ & $\begin{array}{l}\mathrm{Fe}_{2} \mathrm{O}_{3} \\
\text { (wt\%) }\end{array}$ & $\begin{array}{l}\mathrm{MnO} \\
\text { (wt\%) }\end{array}$ & $\begin{array}{l}\mathrm{MgO} \\
\text { (wt\%) }\end{array}$ & $\begin{array}{l}\mathrm{CaO} \\
\text { (wt\%) }\end{array}$ & $\begin{array}{l}\mathrm{Na}_{2} \mathrm{O} \\
(w t \%)\end{array}$ & $\begin{array}{c}\mathrm{K}_{2} \mathrm{O} \\
\text { (wt\%) }\end{array}$ & $\begin{array}{l}\mathrm{P}_{2} \mathrm{O}_{5} \\
\text { (wt\%) }\end{array}$ & Total & $\begin{array}{l}\text { LOI } \\
\text { (wt\%) }\end{array}$ & Mg\# \\
\hline \multicolumn{15}{|l|}{ 309-1256D- } \\
\hline $75 \mathrm{R}-1,116-123$ & 753.05 & 49.58 & 1.44 & 14.34 & 14.09 & 0.22 & 7.36 & 10.77 & 2.73 & 0.04 & 0.11 & 100.67 & 1.69 & 50.8 \\
\hline $78 \mathrm{R}-1,42-50$ & 763.95 & 52.42 & 1.45 & 13.74 & 12.20 & 0.24 & 6.89 & 10.70 & 2.87 & 0.07 & 0.11 & 100.69 & 0.21 & 52.8 \\
\hline $78 \mathrm{R}-2,20-33$ & 764.97 & 49.55 & 1.43 & 13.85 & 13.60 & 0.23 & 7.52 & 11.42 & 2.52 & 0.03 & 0.11 & 100.25 & 0.74 & 52.2 \\
\hline $78 R-3,63-76$ & 766.55 & 54.14 & 2.12 & 12.76 & 13.44 & 0.28 & 5.72 & 9.35 & 2.92 & 0.21 & 0.18 & 101.13 & 0.62 & 45.7 \\
\hline $78 \mathrm{R}-3,63-76$ & 766.55 & 52.50 & 2.11 & 12.83 & 14.93 & 0.32 & 5.77 & 9.38 & 3.15 & 0.37 & 0.18 & 101.55 & 0.33 & 43.3 \\
\hline 79R-1, 98-107 & 770.72 & 52.00 & 2.17 & 13.38 & 14.93 & 0.28 & 5.47 & 9.08 & 3.19 & 0.66 & 0.19 & 101.34 & 0.73 & 42.0 \\
\hline $80 \mathrm{R}-2,92-102$ & 781.47 & 51.88 & 1.86 & 14.85 & 12.30 & 0.23 & 5.49 & 10.09 & 2.97 & 1.12 & 0.21 & 100.99 & 1.27 & 46.9 \\
\hline $82 \mathrm{R}-2,10-20$ & 793.77 & 52.04 & 1.36 & 13.65 & 11.88 & 0.22 & 7.14 & 10.98 & 2.54 & 0.24 & 0.11 & 100.15 & 0.46 & 54.3 \\
\hline $84 \mathrm{R}-2,61-70$ & 803.78 & 52.51 & 1.08 & 14.21 & 11.02 & 0.18 & 7.87 & 10.77 & 2.22 & 0.06 & 0.10 & 100.01 & 1.18 & 58.6 \\
\hline $85 \mathrm{R}-2,124-136$ & 814.97 & 49.50 & 1.06 & 13.65 & 15.33 & 0.22 & 7.16 & 10.70 & 2.19 & 0.17 & 0.11 & 100.08 & 1.31 & 48.0 \\
\hline $85 \mathrm{R}-2,124-136$ & 814.97 & 53.40 & 1.12 & 14.45 & 10.18 & 0.21 & 7.28 & 10.90 & 2.26 & 0.11 & 0.11 & 100.01 & 1.14 & 58.6 \\
\hline $85 R-4,83-92$ & 816.46 & 51.63 & 1.29 & 14.35 & 11.61 & 0.18 & 7.00 & 10.76 & 2.42 & 0.06 & 0.10 & 99.40 & 1.35 & 54.4 \\
\hline $85 R-5,0-21$ & 816.83 & 51.32 & 1.28 & 14.23 & 11.79 & 0.20 & 7.14 & 11.03 & 2.36 & 0.05 & 0.10 & 99.49 & 0.99 & 54.5 \\
\hline $87 \mathrm{R}-1,22-35$ & 831.06 & 51.12 & 1.32 & 13.66 & 13.06 & 0.20 & 7.33 & 11.35 & 2.34 & 0.05 & 0.10 & 100.52 & 0.63 & 52.6 \\
\hline 87R-2, 83-90 & 832.90 & 50.44 & 1.23 & 14.87 & 11.59 & 0.15 & 7.58 & 11.02 & 2.89 & 0.05 & 0.09 & 99.90 & 1.59 & 56.4 \\
\hline $88 \mathrm{R}-1,10-21$ & 840.41 & 51.19 & 1.29 & 13.88 & 12.90 & 0.19 & 7.34 & 11.40 & 2.37 & 0.05 & 0.10 & 100.71 & 0.38 & 52.9 \\
\hline $88 \mathrm{R}-1,56-64$ & 840.68 & 50.67 & 1.31 & 14.15 & 12.98 & 0.21 & 7.35 & 11.41 & 2.36 & 0.06 & 0.10 & 100.61 & 0.66 & 52.8 \\
\hline $92 \mathrm{R}-1,102-109$ & 869.83 & 50.85 & 1.21 & 14.40 & 10.98 & 0.19 & 8.22 & 11.26 & 2.78 & 0.39 & 0.12 & 100.38 & 0.54 & 59.7 \\
\hline 97R-1, 61-70 & 898.37 & 50.12 & 1.11 & 14.91 & 10.94 & 0.17 & 8.37 & 12.14 & 2.26 & 0.07 & 0.09 & 100.16 & 1.15 & 60.2 \\
\hline $97 \mathrm{R}-1,102-109$ & 898.69 & 49.93 & 1.11 & 14.93 & 10.83 & 0.19 & 8.04 & 12.27 & 2.19 & 0.06 & 0.09 & 99.64 & 1.09 & 59.5 \\
\hline 99R-2, 29-37 & 908.97 & 51.33 & 1.22 & 13.86 & 12.21 & 0.18 & 7.77 & 11.12 & 2.53 & 0.08 & 0.10 & 100.38 & 0.49 & 55.7 \\
\hline $99 \mathrm{R}-2,101-120$ & 909.63 & 50.86 & 1.22 & 13.78 & 12.35 & 0.18 & 7.74 & 11.35 & 2.23 & 0.05 & 0.09 & 99.84 & 0.46 & 55.3 \\
\hline $100 \mathrm{R}-1,136-144$ & 911.71 & 49.93 & 1.22 & 14.03 & 12.47 & 0.18 & 8.18 & 11.75 & 2.12 & 0.03 & 0.10 & 99.99 & 0.74 & 56.5 \\
\hline $102 \mathrm{R}-1,17-29$ & 926.86 & 50.77 & 1.33 & 13.79 & 12.70 & 0.19 & 7.51 & 11.26 & 2.19 & 0.06 & 0.11 & 99.90 & 0.56 & 53.9 \\
\hline $102 \mathrm{R}-1,128-137$ & 927.63 & 50.88 & 1.21 & 14.45 & 11.26 & 0.15 & 8.26 & 11.35 & 2.05 & 0.05 & 0.10 & 99.75 & 2.21 & 59.2 \\
\hline $103 \mathrm{R}-1,20-28$ & 936.21 & 49.99 & 1.33 & 13.90 & 12.44 & 0.23 & 8.51 & 11.55 & 2.26 & 0.03 & 0.11 & 100.36 & 0.79 & 57.5 \\
\hline $105 \mathrm{R}-1,35-46$ & 945.99 & 50.96 & 1.36 & 13.78 & 12.91 & 0.21 & 7.43 & 11.24 & 2.30 & 0.05 & 0.11 & 100.36 & 0.49 & 53.3 \\
\hline 107R-1, 9-17 & 955.33 & 52.13 & 1.34 & 13.52 & 12.56 & 0.24 & 7.38 & 10.75 & 2.30 & 0.05 & 0.11 & 100.38 & 0.64 & 53.8 \\
\hline $108 \mathrm{R}-1,38-48$ & 959.21 & 50.76 & 1.24 & 13.93 & 11.57 & 0.21 & 8.13 & 11.52 & 2.25 & 0.05 & 0.10 & 99.76 & 0.84 & 58.2 \\
\hline $108 \mathrm{R}-2,52-61$ & 960.51 & 50.83 & 1.52 & 14.79 & 11.48 & 0.16 & 7.84 & 10.75 & 2.56 & 0.08 & 0.13 & 100.15 & 1.84 & 57.5 \\
\hline $109 \mathrm{R}-1,117-129$ & 965.93 & 51.34 & 1.34 & 13.42 & 13.09 & 0.20 & 7.12 & 10.77 & 2.28 & 0.04 & 0.11 & 99.72 & 0.55 & 51.8 \\
\hline $110 \mathrm{R}-1,26-32$ & 969.91 & 51.05 & 1.36 & 13.47 & 13.28 & 0.21 & 7.20 & 10.78 & 2.33 & 0.05 & 0.12 & 99.82 & 0.55 & 51.7 \\
\hline $110 \mathrm{R}-1,130-144$ & 970.89 & 50.83 & 1.19 & 13.46 & 13.24 & 0.17 & 7.34 & 11.04 & 2.03 & 0.05 & 0.10 & 99.44 & 1.65 & 52.3 \\
\hline $110 \mathrm{R}-2,76-88$ & 971.71 & 50.96 & 1.33 & 13.80 & 12.62 & 0.20 & 7.55 & 11.30 & 2.23 & 0.06 & 0.11 & 100.15 & 0.53 & 54.2 \\
\hline $111 \mathrm{R}-1,26-32$ & 974.68 & 50.09 & 2.35 & 12.27 & 17.32 & 0.28 & 5.86 & 9.71 & 2.62 & 0.08 & 0.21 & 100.78 & 0.36 & 40.1 \\
\hline $111 \mathrm{R}-1,48-55$ & 974.92 & 53.34 & 1.31 & 13.40 & 12.32 & 0.16 & 6.67 & 9.79 & 2.25 & 0.04 & 0.11 & 99.39 & 1.33 & 51.7 \\
\hline $112 \mathrm{R}-1,113-122$ & 980.26 & 51.23 & 1.10 & 14.02 & 11.98 & 0.22 & 7.76 & 11.38 & 2.00 & 0.03 & 0.10 & 99.82 & 0.73 & 56.2 \\
\hline $113 R-1,43-53$ & 984.58 & 51.17 & 1.20 & 13.80 & 12.25 & 0.26 & 7.84 & 11.39 & 1.97 & 0.03 & 0.10 & 100.01 & 0.45 & 55.9 \\
\hline $114 \mathrm{R}-1,117-122$ & 989.87 & 50.15 & 1.21 & 14.75 & 12.20 & 0.17 & 7.95 & 11.48 & 2.20 & 0.05 & 0.11 & 100.26 & 0.73 & 56.3 \\
\hline $114 \mathrm{R}-1,127-142$ & 990.04 & 50.83 & 1.16 & 14.13 & 12.16 & 0.17 & 7.88 & 11.26 & 2.06 & 0.05 & 0.10 & 99.80 & 0.96 & 56.2 \\
\hline $115 \mathrm{R}-1,40-50$ & 994.01 & 51.46 & 1.35 & 13.41 & 13.26 & 0.20 & 7.07 & 10.72 & 2.25 & 0.09 & 0.12 & 99.93 & 0.60 & 51.3 \\
\hline $116 \mathrm{R}-1,0-15$ & 998.40 & 51.75 & 1.36 & 13.48 & 12.98 & 0.20 & 7.09 & 10.62 & 2.22 & 0.09 & 0.12 & 99.91 & 0.49 & 51.9 \\
\hline $117 \mathrm{R}-1,56-67$ & 1003.85 & 51.23 & 1.22 & 13.83 & 12.04 & 0.23 & 8.16 & 11.44 & 2.05 & 0.03 & 0.10 & 100.33 & 0.96 & 57.3 \\
\hline 117R-1, 97-107 & 1004.17 & 51.05 & 1.15 & 14.12 & 12.22 & 0.16 & 8.23 & 10.61 & 1.72 & 0.04 & 0.10 & 99.40 & 2.45 & 57.1 \\
\hline $118 \mathrm{R}-1,48-56$ & 1008.63 & 51.09 & 1.20 & 13.97 & 12.41 & 0.20 & 8.27 & 11.27 & 1.87 & 0.03 & 0.10 & 100.42 & 1.26 & 56.9 \\
\hline $120 \mathrm{R}-1,95-105$ & 1018.42 & 51.45 & 1.36 & 13.39 & 13.18 & 0.23 & 7.06 & 10.68 & 2.28 & 0.06 & 0.12 & 99.81 & 0.46 & 51.4 \\
\hline $122 \mathrm{R}-1,28-33$ & 1027.55 & 55.63 & 1.26 & 9.71 & 16.05 & 0.17 & 6.25 & 5.18 & 2.58 & 0.07 & 0.10 & 96.99 & 5.87 & 43.5 \\
\hline $122 \mathrm{R}-1,67-79$ & 1027.97 & 39.32 & 1.15 & 9.87 & 34.55 & 0.18 & 6.49 & 4.28 & 2.95 & 0.05 & 0.11 & 98.94 & 1.80 & 27.1 \\
\hline 126R-1，39-47 & 1046.95 & 49.93 & 1.47 & 13.41 & 12.97 & 0.20 & 7.40 & 11.39 & 2.13 & 0.03 & 0.12 & 99.05 & 1.37 & 53.0 \\
\hline 128R-1, 80-91 & 1056.77 & 53.21 & 1.43 & 12.07 & 12.78 & 0.20 & 8.39 & 7.97 & 4.63 & 0.05 & 0.11 & 100.85 & 1.38 & 56.5 \\
\hline $129 \mathrm{R}-1,34-51$ & 1061.51 & 50.78 & 1.54 & 13.78 & 12.60 & 0.23 & 7.06 & 11.73 & 2.26 & 0.03 & 0.14 & 100.15 & 1.01 & 52.6 \\
\hline $129 \mathrm{R}-1,125-139$ & 1062.26 & 50.06 & 1.51 & 13.78 & 12.66 & 0.18 & 6.97 & 11.81 & 2.32 & 0.03 & 0.14 & 99.47 & 1.53 & 52.1 \\
\hline 130R-1, 14-22 & 1065.84 & 50.81 & 1.32 & 13.20 & 12.95 & 0.20 & 6.79 & 10.95 & 2.45 & 0.04 & 0.12 & 98.82 & 0.77 & 50.9 \\
\hline 130R-2, 21-34 & 1067.28 & 51.89 & 1.22 & 13.62 & 12.02 & 0.20 & 7.86 & 9.43 & 3.04 & 0.21 & 0.11 & 99.58 & 2.44 & 56.4 \\
\hline 130R-2, 79-88 & 1067.78 & 51.26 & 1.34 & 13.34 & 13.10 & 0.21 & 6.74 & 11.02 & 2.34 & 0.03 & 0.12 & 99.50 & 0.72 & 50.5 \\
\hline $131 \mathrm{R}-1,65-79$ & 1071.24 & 51.48 & 1.32 & 13.58 & 12.99 & 0.20 & 6.82 & 11.29 & 2.29 & 0.04 & 0.12 & 100.11 & 1.30 & 51.0 \\
\hline $131 \mathrm{R}-1,137-147$ & 1071.86 & 51.44 & 1.32 & 13.48 & 12.94 & 0.21 & 6.88 & 11.24 & 2.27 & 0.04 & 0.11 & 99.93 & 1.28 & 51.3 \\
\hline $132 \mathrm{R}-1,0-18$ & 1075.30 & 51.15 & 1.29 & 13.64 & 12.63 & 0.18 & 7.08 & 11.37 & 2.23 & 0.04 & 0.11 & 99.71 & 1.72 & 52.6 \\
\hline $133 \mathrm{R}-1,42-52$ & 1082.15 & 50.82 & 1.25 & 14.47 & 11.44 & 0.24 & 8.07 & 12.20 & 2.14 & 0.02 & 0.10 & 100.74 & 1.42 & 58.3 \\
\hline $133 \mathrm{R}-1,141-148$ & 1082.41 & 51.65 & 1.06 & 13.78 & 12.07 & 0.20 & 7.77 & 10.84 & 2.37 & 0.03 & 0.10 & 99.87 & 1.57 & 56.0 \\
\hline $134 \mathrm{R}-2,40-52$ & 1086.54 & 51.87 & 1.58 & 13.61 & 12.78 & 0.29 & 7.63 & 10.26 & 3.20 & 0.10 & 0.14 & 101.45 & 2.04 & 54.2 \\
\hline $135 \mathrm{R}-1,54-64$ & 1090.56 & 51.71 & 1.66 & 12.96 & 14.69 & 0.28 & 6.65 & 9.85 & 2.87 & 0.06 & 0.14 & 100.86 & 1.55 & 47.3 \\
\hline $135 \mathrm{R}-1,121-127$ & 1090.86 & 55.49 & 1.38 & 11.55 & 15.74 & 0.27 & 6.38 & 6.27 & 2.53 & 0.02 & 0.12 & 99.75 & 4.71 & 44.5 \\
\hline 136R-1, 11-16 & 1094.65 & 50.69 & 1.63 & 13.36 & 12.99 & 0.23 & 7.78 & 10.79 & 3.43 & 0.12 & 0.16 & 101.18 & 1.79 & 54.2 \\
\hline $136 \mathrm{R}-1,82-90$ & 1095.36 & 50.33 & 1.11 & 14.62 & 11.18 & 0.26 & 9.34 & 11.10 & 3.00 & 0.10 & 0.08 & 101.12 & 2.04 & 62.3 \\
\hline 137R-1, 92-99 & 1100.11 & 52.88 & 1.33 & 13.44 & 12.08 & 0.32 & 8.42 & 9.73 & 3.43 & 0.13 & 0.10 & 101.86 & 2.79 & 58.0 \\
\hline $138 \mathrm{R}-2,32-41$ & 1105.89 & 50.89 & 1.32 & 13.77 & 12.73 & 0.21 & 7.56 & 11.39 & 2.16 & 0.04 & 0.10 & 100.18 & 1.33 & 54.0 \\
\hline 139R-1, 93-99 & 1109.83 & 52.05 & 1.62 & 13.44 & 12.92 & 0.34 & 7.87 & 9.41 & 3.26 & 0.08 & 0.15 & 101.12 & 2.79 & 54.7 \\
\hline
\end{tabular}


Table T1 (continued). (Continued on next page.)

\begin{tabular}{|c|c|c|c|c|c|c|c|c|c|c|c|c|c|c|}
\hline $\begin{array}{l}\text { Core, section, } \\
\text { interval }(\mathrm{cm})\end{array}$ & $\begin{array}{l}\text { Depth } \\
\text { (mbsf) }\end{array}$ & $\begin{array}{c}\mathrm{SiO}_{2} \\
(\mathrm{wt} \%)\end{array}$ & $\begin{array}{c}\mathrm{TiO}_{2} \\
\text { (wt\%) }\end{array}$ & $\begin{array}{l}\mathrm{Al}_{2} \mathrm{O}_{3} \\
\text { (wt\%) }\end{array}$ & $\begin{array}{l}\mathrm{Fe}_{2} \mathrm{O}_{3} \\
\text { (wt\%) }\end{array}$ & $\begin{array}{l}\mathrm{MnO} \\
(\mathrm{wt} \%)\end{array}$ & $\begin{array}{l}\mathrm{MgO} \\
\text { (wt\%) }\end{array}$ & $\begin{array}{c}\mathrm{CaO} \\
(\mathrm{wt} \%)\end{array}$ & $\begin{array}{l}\mathrm{Na}_{2} \mathrm{O} \\
(w t \%)\end{array}$ & $\begin{array}{c}\mathrm{K}_{2} \mathrm{O} \\
\text { (wt\%) }\end{array}$ & $\begin{array}{l}\mathrm{P}_{2} \mathrm{O}_{5} \\
\text { (wt\%) }\end{array}$ & Total & $\begin{array}{c}\text { LOI } \\
\text { (wt\%) }\end{array}$ & Mg\# \\
\hline $140 R-1,55-65$ & 1114.72 & 51.62 & 1.64 & 13.99 & 14.82 & 0.26 & 7.87 & 6.90 & 3.58 & 0.06 & 0.16 & 100.91 & 4.09 & 51.2 \\
\hline $142 \mathrm{R}-1,38-48$ & 1123.75 & 49.98 & 1.37 & 14.32 & 11.19 & 0.22 & 9.06 & 11.74 & 2.32 & 0.12 & 0.13 & 100.45 & 4.45 & 61.6 \\
\hline $142 \mathrm{R}-2,40-55$ & 1125.34 & 49.75 & 1.33 & 15.10 & 11.14 & 0.20 & 8.62 & 11.28 & 3.06 & 0.06 & 0.13 & 100.67 & 2.43 & 60.5 \\
\hline 143R-1, 72-82 & 1128.71 & 50.63 & 1.16 & 13.87 & 12.29 & 0.19 & 8.04 & 11.17 & 2.45 & 0.02 & 0.09 & 99.90 & 1.73 & 56.4 \\
\hline $143 R-1,122-125$ & 1129.23 & 50.37 & 1.21 & 13.79 & 12.56 & 0.19 & 7.87 & 11.24 & 2.24 & 0.02 & 0.09 & 99.58 & 1.75 & 55.4 \\
\hline $143 R-2,40-49$ & 1129.64 & 50.93 & 1.12 & 14.14 & 12.13 & 0.21 & 7.65 & 11.43 & 2.07 & 0.03 & 0.10 & 99.80 & 1.45 & 55.5 \\
\hline $144 R-2,39-56$ & 1134.38 & 50.92 & 1.15 & 14.13 & 12.14 & 0.20 & 7.65 & 11.45 & 2.06 & 0.03 & 0.10 & 99.82 & 1.46 & 55.5 \\
\hline $145 R-2,73-89$ & 1139.63 & 51.37 & 1.06 & 14.00 & 11.46 & 0.20 & 8.46 & 11.08 & 2.25 & 0.03 & 0.09 & 100.00 & 1.51 & 59.4 \\
\hline $146 \mathrm{R}-1,30-54$ & 1142.50 & 51.52 & 1.03 & 14.04 & 11.53 & 0.19 & 8.06 & 11.20 & 2.19 & 0.04 & 0.09 & 99.89 & 1.95 & 58.1 \\
\hline $146 \mathrm{R}-2,80-88$ & 1143.86 & 50.75 & 1.05 & 14.13 & 11.71 & 0.19 & 7.81 & 11.37 & 2.14 & 0.04 & 0.10 & 99.30 & 1.87 & 56.9 \\
\hline $147 \mathrm{R}-1,40-48$ & 1145.98 & 50.92 & 1.31 & 13.64 & 13.09 & 0.21 & 7.22 & 10.46 & 3.07 & 0.08 & 0.10 & 100.09 & 2.13 & 52.2 \\
\hline 149R-1, 57-63 & 1156.66 & 52.49 & 1.90 & 12.27 & 15.17 & 0.24 & 6.81 & 8.27 & 3.65 & 0.06 & 0.16 & 101.03 & 1.58 & 47.0 \\
\hline 149R-1, 138-145 & 1157.33 & 52.59 & 1.85 & 12.48 & 15.15 & 0.28 & 6.73 & 8.82 & 2.73 & 0.05 & 0.15 & 100.83 & 1.83 & 46.8 \\
\hline 151R-1, 119-125 & 1166.43 & 51.75 & 1.58 & 13.26 & 14.35 & 0.29 & 6.85 & 10.32 & 2.56 & 0.04 & 0.13 & 101.11 & 1.23 & 48.6 \\
\hline $153 R-2,36-47$ & 1176.70 & 51.50 & 1.68 & 13.02 & 14.80 & 0.24 & 6.41 & 10.03 & 2.58 & 0.03 & 0.14 & 100.43 & 0.98 & 46.2 \\
\hline $153 R-2,103-115$ & 1177.28 & 52.02 & 1.54 & 12.98 & 13.61 & 0.26 & 7.11 & 9.94 & 2.80 & 0.06 & 0.12 & 100.44 & 1.37 & 50.8 \\
\hline $154 R-2,30-52$ & 1181.45 & 52.13 & 1.68 & 12.48 & 14.26 & 0.27 & 6.94 & 9.80 & 2.80 & 0.11 & 0.13 & 100.60 & 2.10 & 49.1 \\
\hline $155 R-2,60-80$ & 1186.47 & 51.34 & 1.47 & 13.32 & 13.65 & 0.25 & 7.31 & 10.29 & 2.35 & 0.07 & 0.12 & 100.17 & 1.39 & 51.5 \\
\hline 155R-3, 0-10 & 1187.12 & 51.80 & 1.49 & 13.35 & 13.51 & 0.31 & 7.37 & 10.43 & 2.02 & 0.04 & 0.12 & 100.44 & 2.77 & 51.9 \\
\hline 156R-1, 90-100 & 1190.51 & 50.87 & 1.13 & 14.02 & 11.91 & 0.23 & 8.34 & 11.41 & 2.17 & 0.05 & 0.09 & 100.22 & 1.60 & 58.1 \\
\hline $157 R-1,111-125$ & 1195.29 & 51.52 & 1.78 & 12.90 & 15.00 & 0.25 & 6.25 & 10.07 & 2.46 & 0.04 & 0.14 & 100.41 & 1.49 & 45.2 \\
\hline 159R-1, 3-13 & 1203.83 & 51.39 & 1.61 & 13.07 & 14.38 & 0.25 & 6.79 & 10.09 & 2.53 & 0.04 & 0.13 & 100.27 & 1.54 & 48.3 \\
\hline 159R-1, 60-70 & 1204.40 & 52.01 & 1.64 & 11.65 & 14.13 & 0.26 & 7.84 & 10.50 & 2.52 & 0.07 & 0.13 & 100.75 & 3.52 & 52.3 \\
\hline 159R-1, 98-110 & 1204.63 & 50.79 & 1.51 & 13.74 & 13.20 & 0.25 & 7.76 & 10.86 & 2.25 & 0.04 & 0.12 & 100.51 & 2.04 & 53.8 \\
\hline 160R-1, 85-100 & 1209.69 & 51.27 & 1.16 & 13.60 & 12.54 & 0.20 & 7.41 & 11.24 & 2.19 & 0.08 & 0.10 & 99.80 & 1.80 & 53.9 \\
\hline $161 \mathrm{R}-1,105-113$ & 1211.15 & 50.44 & 0.98 & 14.39 & 10.52 & 0.19 & 9.77 & 11.50 & 2.80 & 0.41 & 0.07 & 101.07 & 2.74 & 64.8 \\
\hline $162 R-1,37-45$ & 1213.95 & 50.51 & 1.32 & 14.22 & 11.59 & 0.19 & 7.90 & 11.53 & 2.22 & 0.14 & 0.11 & 99.71 & 2.03 & 57.4 \\
\hline 162R-2, 89-106 & 1215.60 & 50.53 & 1.12 & 14.29 & 11.41 & 0.19 & 8.59 & 11.93 & 1.88 & 0.04 & 0.08 & 100.05 & 1.73 & 59.8 \\
\hline $163 R-2,55-63$ & 1219.91 & 50.84 & 1.49 & 13.49 & 12.99 & 0.23 & 7.56 & 10.89 & 2.23 & 0.05 & 0.12 & 99.87 & 1.86 & 53.5 \\
\hline $164 \mathrm{R}-1,27-50$ & 1223.80 & 50.31 & 1.40 & 14.21 & 12.12 & 0.19 & 7.43 & 11.89 & 2.15 & 0.05 & 0.11 & 99.85 & 1.71 & 54.8 \\
\hline $164 R-2,5-15$ & 1224.49 & 50.58 & 1.40 & 14.18 & 12.18 & 0.20 & 7.37 & 11.92 & 2.12 & 0.03 & 0.11 & 100.09 & 1.43 & 54.5 \\
\hline $164 \mathrm{R}-2,101-107$ & 1225.18 & 51.88 & 1.59 & 13.00 & 14.10 & 0.21 & 6.65 & 10.22 & 2.40 & 0.05 & 0.12 & 100.23 & 1.54 & 48.3 \\
\hline $164 R-3,36-44$ & 1225.86 & 51.37 & 1.77 & 13.09 & 14.86 & 0.25 & 6.59 & 10.16 & 2.45 & 0.03 & 0.14 & 100.72 & 1.54 & 46.7 \\
\hline $165 \mathrm{R}-1,23-33$ & 1228.12 & 52.32 & 1.56 & 12.83 & 13.41 & 0.25 & 7.49 & 9.62 & 2.57 & 0.05 & 0.12 & 100.22 & 1.99 & 52.5 \\
\hline 165R-2, 71-91 & 1230.17 & 51.21 & 1.61 & 12.90 & 14.38 & 0.23 & 6.51 & 10.11 & 2.37 & 0.04 & 0.13 & 99.49 & 1.42 & 47.3 \\
\hline $165 \mathrm{R}-3,19-30$ & 1230.19 & 51.94 & 1.63 & 12.90 & 13.56 & 0.23 & 6.65 & 10.42 & 2.70 & 0.07 & 0.13 & 100.23 & 1.64 & 49.2 \\
\hline $166 \mathrm{R}-1,15-27$ & 1232.87 & 51.81 & 1.64 & 12.69 & 14.12 & 0.24 & 7.43 & 8.89 & 2.85 & 0.06 & 0.14 & 99.85 & 2.04 & 51.0 \\
\hline 166R-1, 126-132 & 1233.74 & 51.01 & 1.69 & 12.79 & 14.68 & 0.24 & 6.26 & 10.26 & 2.32 & 0.04 & 0.13 & 99.42 & 1.05 & 45.7 \\
\hline $166 \mathrm{R}-2,88-99$ & 1235.57 & 51.26 & 1.64 & 12.88 & 13.89 & 0.21 & 6.38 & 9.63 & 3.16 & 0.07 & 0.13 & 99.25 & 1.32 & 47.6 \\
\hline $166 \mathrm{R}-3,49-65$ & 1235.75 & 50.93 & 1.67 & 12.43 & 14.74 & 0.23 & 6.23 & 9.53 & 2.50 & 0.08 & 0.13 & 98.46 & 1.72 & 45.5 \\
\hline $167 \mathrm{R}-1,12-23$ & 1236.68 & 50.18 & 1.15 & 14.20 & 11.42 & 0.18 & 8.32 & 12.12 & 1.94 & 0.04 & 0.09 & 99.63 & 1.00 & 59.0 \\
\hline $167 R-2,50-70$ & 1238.63 & 50.31 & 1.08 & 14.06 & 11.29 & 0.18 & 8.47 & 12.12 & 1.91 & 0.06 & 0.08 & 99.56 & 1.42 & 59.7 \\
\hline $168 \mathrm{R}-1,58-72$ & 1243.18 & 50.56 & 1.54 & 13.31 & 13.37 & 0.21 & 6.87 & 11.24 & 2.14 & 0.03 & 0.12 & 99.39 & 0.75 & 50.4 \\
\hline $168 \mathrm{R}-3,23-32$ & 1245.16 & 50.80 & 1.52 & 13.52 & 13.13 & 0.21 & 7.04 & 11.46 & 2.15 & 0.04 & 0.12 & 99.97 & 0.95 & 51.5 \\
\hline $168 R-4,78-90$ & 1247.03 & 50.89 & 1.55 & 13.40 & 13.40 & 0.21 & 6.86 & 11.37 & 2.08 & 0.04 & 0.13 & 99.92 & 1.30 & 50.3 \\
\hline $169 \mathrm{R}-2,40-54$ & 1248.75 & 51.59 & 1.18 & 13.69 & 12.71 & 0.20 & 7.45 & 10.99 & 2.13 & 0.04 & 0.11 & 100.08 & 1.89 & 53.7 \\
\hline $169 \mathrm{R}-3,18-37$ & 1249.88 & 53.19 & 1.01 & 12.95 & 11.91 & 0.19 & 8.09 & 9.65 & 2.63 & 0.08 & 0.08 & 99.78 & 2.49 & 57.3 \\
\hline $170 R-2,40-48$ & 1253.50 & 51.29 & 1.19 & 13.74 & 12.61 & 0.19 & 7.53 & 11.35 & 1.89 & 0.04 & 0.11 & 99.95 & 2.19 & 54.2 \\
\hline 170R-2, 130-144 & 1254.31 & 50.22 & 1.04 & 14.79 & 10.77 & 0.17 & 8.76 & 12.19 & 2.00 & 0.07 & 0.07 & 100.10 & 1.35 & 61.7 \\
\hline $170 R-3,22-40$ & 1254.68 & 50.37 & 1.01 & 14.52 & 10.44 & 0.17 & 9.28 & 11.25 & 2.99 & 0.33 & 0.07 & 100.43 & 2.81 & 63.8 \\
\hline \multicolumn{15}{|l|}{ 312-1256D- } \\
\hline 173R-1, 0-10 & 1260.60 & 51.27 & 1.10 & 13.93 & 12.15 & 0.21 & 7.71 & 11.68 & 1.94 & 0.03 & 0.09 & 100.11 & 1.09 & 55.7 \\
\hline 174R-1, 73-79 & 1266.13 & 51.47 & 1.55 & 12.01 & 14.36 & 0.27 & 6.44 & 8.52 & 2.73 & 0.25 & 0.12 & 97.71 & 1.33 & 47.0 \\
\hline $175 R-1,75-83$ & 1272.05 & 51.19 & 1.53 & 13.73 & 13.48 & 0.22 & 6.82 & 11.18 & 2.24 & 0.05 & 0.13 & 100.56 & 1.04 & 50.0 \\
\hline 176R-1, 116-122 & 1277.27 & 50.54 & 1.76 & 13.07 & 15.06 & 0.31 & 6.30 & 10.32 & 2.36 & 0.08 & 0.14 & 99.94 & 1.42 & 45.3 \\
\hline $176 R-2,42-55$ & 1278.03 & 50.22 & 2.26 & 12.50 & 17.03 & 0.29 & 5.16 & 9.33 & 2.54 & 0.05 & 0.19 & 99.55 & 1.00 & 37.5 \\
\hline $181 \mathrm{R}-1,51-56$ & 1300.61 & 50.02 & 1.22 & 13.81 & 12.47 & 0.22 & 7.61 & 11.50 & 2.20 & 0.08 & 0.09 & 99.21 & 0.73 & 54.7 \\
\hline $182 \mathrm{R}-1,18-25$ & 1305.09 & 50.60 & 1.22 & 14.03 & 12.67 & 0.21 & 7.55 & 11.37 & 2.26 & 0.07 & 0.09 & 100.06 & 0.28 & 54.1 \\
\hline 184R-1, 0-8 & 1314.50 & 51.02 & 1.52 & 13.23 & 14.03 & 0.22 & 6.71 & 10.37 & 2.64 & 0.06 & 0.12 & 99.91 & 0.45 & 48.6 \\
\hline $186 \mathrm{R}-1,12-20$ & 1320.10 & 51.26 & 1.43 & 13.33 & 12.74 & 0.19 & 7.50 & 10.09 & 3.04 & 0.18 & 0.11 & 99.85 & 0.59 & 53.8 \\
\hline $187 \mathrm{R}-2,21-31$ & 1325.88 & 50.79 & 1.42 & 13.37 & 13.67 & 0.20 & 6.84 & 9.78 & 3.14 & 0.26 & 0.11 & 99.57 & 0.39 & 49.8 \\
\hline 189R-1, 0-13 & 1333.90 & 49.71 & 1.40 & 15.22 & 10.63 & 0.19 & 8.25 & 11.03 & 2.87 & 0.27 & 0.14 & 99.70 & 0.53 & 60.6 \\
\hline 189R-1, 71-89 & 1334.61 & 49.00 & 1.41 & 15.57 & 10.43 & 0.19 & 8.39 & 12.12 & 2.45 & 0.08 & 0.14 & 99.77 & 0.64 & 61.4 \\
\hline 196R-1, 16-20 & 1363.86 & 50.84 & 1.37 & 14.10 & 12.71 & 0.18 & 7.41 & 11.47 & 2.41 & 0.06 & 0.11 & 100.65 & 0.22 & 53.6 \\
\hline $202 \mathrm{R}-1,25-37$ & 1373.05 & 50.25 & 1.90 & 12.85 & 15.90 & 0.23 & 6.01 & 9.56 & 2.50 & 0.09 & 0.14 & 99.42 & 0.00 & 42.8 \\
\hline 205R-1, 0-4 & 1382.10 & 50.48 & 1.81 & 13.03 & 14.57 & 0.22 & 6.78 & 10.44 & 2.19 & 0.05 & 0.14 & 99.69 & 0.43 & 47.9 \\
\hline 206R-1, 8-12 & 1386.98 & 50.79 & 1.41 & 13.41 & 13.57 & 0.21 & 7.11 & 10.46 & 2.63 & 0.05 & 0.11 & 99.73 & 0.00 & 50.9 \\
\hline $212 \mathrm{R}-1,4-7$ & 1404.14 & 50.01 & 1.50 & 13.60 & 13.03 & 0.20 & 7.06 & 11.19 & 2.23 & 0.06 & 0.12 & 98.99 & -0.17 & 51.7 \\
\hline $214 \mathrm{R}-1,26-35$ & 1411.16 & 51.46 & 3.93 & 12.16 & 17.51 & 0.15 & 3.61 & 6.97 & 3.46 & 0.21 & 0.37 & 99.82 & 0.57 & 29.0 \\
\hline
\end{tabular}


Table T1 (continued).

\begin{tabular}{|c|c|c|c|c|c|c|c|c|c|c|c|c|c|c|}
\hline $\begin{array}{l}\text { Core, section, } \\
\text { interval }(\mathrm{cm})\end{array}$ & $\begin{array}{l}\text { Depth } \\
\text { (mbsf) }\end{array}$ & $\begin{array}{c}\mathrm{SiO}_{2} \\
(\mathrm{wt} \%)\end{array}$ & $\begin{array}{c}\mathrm{TiO}_{2} \\
\text { (wt\%) }\end{array}$ & $\begin{array}{l}\mathrm{Al}_{2} \mathrm{O}_{3} \\
\text { (wt\%) }\end{array}$ & $\begin{array}{l}\mathrm{Fe}_{2} \mathrm{O}_{3} \\
\text { (wt\%) }\end{array}$ & $\begin{array}{l}\mathrm{MnO} \\
(\mathrm{wt} \%)\end{array}$ & $\begin{array}{l}\mathrm{MgO} \\
(\mathrm{wt} \%)\end{array}$ & $\begin{array}{c}\mathrm{CaO} \\
(\mathrm{wt} \%)\end{array}$ & $\begin{array}{l}\mathrm{Na}_{2} \mathrm{O} \\
(w t \%)\end{array}$ & $\begin{array}{c}\mathrm{K}_{2} \mathrm{O} \\
(\mathrm{wt} \%)\end{array}$ & $\begin{array}{l}\mathrm{P}_{2} \mathrm{O}_{5} \\
\text { (wt\%) }\end{array}$ & Total & $\begin{array}{c}\text { LOI } \\
\text { (wt\%) }\end{array}$ & Mg\# \\
\hline $214 \mathrm{R}-1,26-35$ & 1411.16 & 49.81 & 1.34 & 14.68 & 11.38 & 0.16 & 7.82 & 11.86 & 2.65 & 0.15 & 0.11 & 99.95 & 0.66 & 57.6 \\
\hline $214 \mathrm{R}-1,52-58$ & 1411.42 & 52.58 & 3.60 & 12.35 & 16.67 & 0.16 & 3.53 & 6.50 & 3.41 & 0.15 & 0.40 & 99.36 & 1.43 & 29.6 \\
\hline 214R-2, 119-129 & 1413.55 & 49.49 & 1.15 & 15.49 & 10.24 & 0.13 & 7.22 & 12.48 & 2.85 & 0.08 & 0.08 & 99.21 & 0.46 & 58.2 \\
\hline $215 R-1,43-49$ & 1416.13 & 49.70 & 1.18 & 15.59 & 10.74 & 0.14 & 7.38 & 12.03 & 2.70 & 0.07 & 0.08 & 99.60 & 0.47 & 57.6 \\
\hline $216 \mathrm{R}-1,49-58$ & 1418.39 & 50.24 & 0.94 & 15.39 & 9.81 & 0.14 & 7.87 & 12.72 & 2.49 & 0.08 & 0.06 & 99.76 & 0.34 & 61.4 \\
\hline $217 \mathrm{R}-1,17-21$ & 1421.77 & 49.42 & 0.70 & 15.68 & 8.08 & 0.12 & 9.23 & 13.78 & 2.20 & 0.06 & 0.05 & 99.31 & 0.71 & 69.3 \\
\hline $217 \mathrm{R}-1,29-33$ & 1421.89 & 49.13 & 0.80 & 15.62 & 9.47 & 0.13 & 8.85 & 12.68 & 2.41 & 0.07 & 0.05 & 99.20 & 0.60 & 64.9 \\
\hline $219 \mathrm{R}-1,25-32$ & 1430.26 & 50.48 & 0.98 & 14.96 & 9.40 & 0.16 & 8.37 & 13.33 & 2.19 & 0.09 & 0.08 & 100.02 & 0.34 & 63.8 \\
\hline 220R-1, 92-100 & 1435.92 & 49.82 & 1.11 & 14.46 & 10.76 & 0.16 & 8.30 & 11.95 & 2.61 & 0.08 & 0.08 & 99.33 & 0.67 & 60.4 \\
\hline $222 \mathrm{R}-2,25-35$ & 1446.37 & 48.99 & 0.76 & 15.07 & 8.88 & 0.13 & 9.45 & 12.94 & 2.37 & 0.07 & 0.07 & 98.72 & 0.61 & 67.8 \\
\hline 223R-1, 137-144 & 1450.68 & 48.72 & 0.89 & 14.11 & 10.11 & 0.15 & 10.65 & 11.85 & 2.26 & 0.07 & 0.08 & 98.88 & 1.66 & 67.6 \\
\hline $223 R-3,12-24$ & 1452.40 & 48.22 & 0.65 & 15.07 & 9.59 & 0.13 & 9.52 & 12.86 & 2.22 & 0.06 & 0.05 & 98.38 & 0.60 & 66.3 \\
\hline 227R-1, 50-61 & 1469.00 & 49.44 & 1.41 & 13.36 & 14.09 & 0.20 & 7.49 & 10.92 & 2.54 & 0.05 & 0.10 & 99.59 & 0.25 & 51.3 \\
\hline $227 R-1,80-86$ & 1469.30 & 48.74 & 1.55 & 13.83 & 14.32 & 0.21 & 7.13 & 11.69 & 2.48 & 0.06 & 0.09 & 100.11 & 0.93 & 49.6 \\
\hline 227R-1, 113-126 & 1469.63 & 50.19 & 1.36 & 13.52 & 12.67 & 0.15 & 7.64 & 11.16 & 2.87 & 0.07 & 0.12 & 99.73 & 0.45 & 54.4 \\
\hline $230 \mathrm{R}-1,72-83$ & 1483.72 & 47.45 & 1.72 & 13.12 & 15.21 & 0.18 & 7.23 & 11.04 & 2.50 & 0.06 & 0.06 & 98.56 & 0.15 & 48.5 \\
\hline 230R-2, 49-61 & 1484.99 & 49.47 & 1.12 & 14.43 & 10.69 & 0.16 & 8.52 & 12.60 & 2.29 & 0.07 & 0.06 & 99.39 & 0.68 & 61.2 \\
\hline 231R-1, 90-100 & 1488.80 & 49.14 & 1.30 & 14.06 & 10.94 & 0.16 & 8.36 & 12.57 & 2.34 & 0.06 & 0.07 & 98.99 & 0.86 & 60.2 \\
\hline 231R-3, 80-98 & 1491.36 & 49.39 & 1.29 & 14.10 & 11.15 & 0.17 & 8.59 & 12.56 & 2.40 & 0.06 & 0.05 & 99.75 & 0.51 & 60.4 \\
\hline 232R-1, 66-81 & 1493.56 & 49.43 & 1.17 & 14.31 & 11.17 & 0.16 & 8.64 & 12.35 & 2.33 & 0.06 & 0.06 & 99.67 & 0.62 & 60.5 \\
\hline $232 \mathrm{R}-2,35-50$ & 1494.33 & 48.90 & 1.26 & 14.01 & 11.46 & 0.15 & 8.05 & 11.99 & 2.34 & 0.05 & 0.08 & 98.28 & 0.84 & 58.2 \\
\hline 233R-1, 14-19 & 1497.64 & 50.31 & 0.97 & 14.15 & 11.03 & 0.15 & 8.41 & 11.94 & 2.61 & 0.05 & 0.06 & 99.68 & 1.03 & 60.2 \\
\hline 234R-1, 26-29 & 1502.76 & 49.46 & 2.17 & 12.49 & 16.35 & 0.29 & 5.26 & 10.61 & 2.79 & 0.09 & 0.19 & 99.70 & 2.19 & 38.9 \\
\hline
\end{tabular}

Note: $\mathrm{LOI}=$ loss on ignition, $\mathrm{Mg} \#=100 \times \mathrm{Mg} /(\mathrm{Mg}+\mathrm{Fe})$.

Table T2. Measured major element contents and recommended values for reference samples. (See table notes.)

\begin{tabular}{|c|c|c|c|c|c|c|c|c|c|}
\hline \multirow{2}{*}{$\begin{array}{l}\text { Element } \\
\text { oxide } \\
\text { (wt\%) }\end{array}$} & \multicolumn{3}{|c|}{ JB-1b } & \multicolumn{3}{|c|}{ JB-2 } & \multicolumn{3}{|c|}{ JB-3 } \\
\hline & $\begin{array}{c}\mathrm{RV} \\
\text { (wt\%) }\end{array}$ & $\begin{array}{l}\text { This study } \\
\text { (wt\%) }\end{array}$ & $\begin{array}{l}\text { RD } \\
(\%)\end{array}$ & $\begin{array}{c}\text { RV } \\
\text { (wt\%) }\end{array}$ & $\begin{array}{l}\text { This study } \\
\text { (wt\%) }\end{array}$ & $\begin{array}{l}\text { RD } \\
\text { (\%) }\end{array}$ & $\begin{array}{c}\text { RV } \\
\text { (wt\%) }\end{array}$ & $\begin{array}{l}\text { This study } \\
\text { (wt\%) }\end{array}$ & $\begin{array}{l}\text { RD } \\
\text { (\%) }\end{array}$ \\
\hline $\mathrm{SiO}_{2}$ & 52.23 & 52.26 & 0.07 & 52.96 & 53.02 & 0.12 & 50.71 & 50.62 & -0.18 \\
\hline $\mathrm{TiO}_{2}$ & 1.29 & 1.29 & 0.13 & 1.18 & 1.17 & -0.80 & 1.44 & 1.45 & 0.28 \\
\hline $\mathrm{Al}_{2} \mathrm{O}_{3}$ & 14.69 & 14.51 & -1.27 & 14.56 & 14.50 & -0.38 & 17.16 & 17.10 & -0.41 \\
\hline $\mathrm{Fe}_{2} \mathrm{O}_{3}$ & 9.22 & 9.18 & -0.42 & 14.17 & 14.11 & -0.41 & 11.81 & 11.82 & 0.07 \\
\hline $\mathrm{MnO}$ & 0.15 & 0.15 & -3.09 & 0.22 & 0.21 & -1.54 & 0.18 & 0.18 & -0.76 \\
\hline $\mathrm{MgO}$ & 8.32 & 8.37 & 0.67 & 4.59 & 4.61 & 0.36 & 5.14 & 4.98 & -3.24 \\
\hline $\mathrm{CaO}$ & 9.81 & 9.81 & -0.00 & 9.77 & 9.78 & 0.10 & 9.76 & 9.72 & -0.43 \\
\hline $\mathrm{Na}_{2} \mathrm{O}$ & 2.69 & 2.65 & -1.28 & 2.03 & 1.99 & -1.72 & 2.69 & 2.71 & 0.76 \\
\hline $\mathrm{K}_{2} \mathrm{O}$ & 1.35 & 1.35 & 0.08 & 0.42 & 0.41 & -0.90 & 0.79 & 0.78 & -2.01 \\
\hline $\mathrm{P}_{2} \mathrm{O}_{5}$ & 0.26 & 0.27 & 1.38 & 0.10 & 0.10 & -4.67 & 0.30 & 0.30 & -1.42 \\
\hline Total: & 100.00 & 100.96 & & 100.00 & 101.07 & & 100.00 & 100.93 & \\
\hline
\end{tabular}

Notes: Recommended values (RV) for Geological Survey of Japan reference samples. Relative deviation (RD) values, an estimate of accuracy, are calculated between RV and values from this study. RV of JB-1b from Terashima et al. (1998), RV of JB-2 and JB-3 from Imai et al. (1995). 
Table T3. Measured trace element abundances and recommended values for reference samples. (See table notes.)

\begin{tabular}{|c|c|c|c|c|c|c|c|c|}
\hline \multirow[b]{2}{*}{ Element } & \multicolumn{4}{|c|}{ W-2 } & \multicolumn{2}{|c|}{ BAS-206 } & \multicolumn{2}{|c|}{ BAS-312 } \\
\hline & $\begin{array}{c}\mathrm{RV} \\
(\mathrm{ppm})\end{array}$ & $\begin{array}{l}\text { This study } \\
\text { (ppm) }\end{array}$ & $\begin{array}{l}\text { RSD } \\
\text { (\%) }\end{array}$ & $\begin{array}{l}\text { RD } \\
\text { (\%) }\end{array}$ & $\begin{array}{l}\text { This study } \\
\text { (ppm) }\end{array}$ & $\begin{array}{l}\text { RSD } \\
(\%)\end{array}$ & $\begin{array}{l}\text { This study } \\
\text { (ppm) }\end{array}$ & $\begin{array}{l}\text { RSD } \\
(\%)\end{array}$ \\
\hline Sc & 36.2 & 33.7 & 12.2 & -6.8 & 51.7 & 4.7 & 45.8 & 1.6 \\
\hline V & 270 & 272 & 2.5 & 0.7 & 465 & 6.3 & 321 & 4.3 \\
\hline $\mathrm{Cr}$ & 92 & 91.3 & 3.1 & -0.7 & 84.6 & 9.1 & 252 & 6.2 \\
\hline Co & 46 & 43.2 & 6.8 & -6.1 & 55.1 & 2.4 & 46.7 & 1.8 \\
\hline $\mathrm{Ni}$ & 74 & 75.0 & 6.5 & 1.4 & 50.7 & 3.8 & 88.9 & 5.2 \\
\hline $\mathrm{Zn}$ & 77 & 74.6 & 3.2 & -3.2 & 133 & 2.4 & 89.3 & 2.2 \\
\hline Ga & 17.4 & 16.7 & 3.9 & -3.9 & 19.2 & 2.5 & 16.5 & 1.4 \\
\hline $\mathrm{Rb}$ & 20.1 & 19.7 & 3.4 & -2.2 & 2.0 & 4.0 & 0.47 & 1.5 \\
\hline $\mathrm{Sr}$ & 191.8 & 188.0 & 3.4 & -2.0 & 102 & 1.4 & 101 & 1.2 \\
\hline$Y$ & 22.8 & 22.8 & 2.3 & -0.01 & 50.2 & 1.7 & 30.8 & 1.5 \\
\hline $\mathrm{Zr}$ & 92.0 & 93.0 & 2.4 & 1.1 & 128 & 1.2 & 79.2 & 2.1 \\
\hline $\mathrm{Zr}^{*}$ & & 101 & 2.4 & 10.1 & & & & \\
\hline $\mathrm{Nb}$ & 7.76 & 7.67 & 2.2 & -1.2 & 4.7 & 1.4 & 3.33 & 1.2 \\
\hline Cs & 0.916 & 0.913 & 3.2 & -0.3 & 0.1 & 4.4 & 0.02 & 5.7 \\
\hline $\mathrm{Ba}$ & 171 & 169 & 1.7 & -1.2 & 42.2 & 5.5 & 12.2 & 3.0 \\
\hline La & 10.59 & 10.4 & 1.4 & -1.5 & 4.7 & 1.0 & 3.03 & 1.0 \\
\hline $\mathrm{Ce}$ & 23.08 & 22.8 & 1.5 & -1.1 & 13.9 & 1.6 & 8.63 & 1.4 \\
\hline $\mathrm{Pr}$ & 3.03 & 3.02 & 1.6 & -0.3 & 2.4 & 1.6 & 1.44 & 2.4 \\
\hline $\mathrm{Nd}$ & 12.95 & 12.9 & 2.4 & -0.0 & 13.2 & 1.3 & 7.83 & 2.9 \\
\hline $\mathrm{Sm}$ & 3.31 & 3.24 & 2.8 & -2.2 & 4.7 & 2.2 & 2.80 & 2.8 \\
\hline $\mathrm{Eu}$ & 1.093 & 1.05 & 4.5 & -3.8 & 1.6 & 0.8 & 1.03 & 3.4 \\
\hline$G d$ & 3.69 & 3.65 & 2.6 & -1.1 & 6.5 & 1.7 & 3.90 & 2.0 \\
\hline $\mathrm{Tb}$ & 0.622 & 0.61 & 3.0 & -1.4 & 1.2 & 2.3 & 0.71 & 2.3 \\
\hline Dy & 3.79 & 3.77 & 3.1 & -0.6 & 7.9 & 1.0 & 4.81 & 0.8 \\
\hline Ho & 0.798 & 0.78 & 2.1 & -2.6 & 1.8 & 1.9 & 1.06 & 2.9 \\
\hline Er & 2.26 & 2.22 & 3.6 & -1.6 & 5.2 & 1.3 & 3.11 & 2.1 \\
\hline $\mathrm{Yb}$ & 2.03 & 2.04 & 2.9 & 0.3 & 4.7 & 1.5 & 3.07 & 2.2 \\
\hline Lu & 0.299 & 0.304 & 3.8 & 1.8 & 0.71 & 1.1 & 0.47 & 1.3 \\
\hline $\mathrm{Hf}$ & 2.30 & 2.33 & 2.3 & 1.3 & 3.3 & 2.5 & 2.02 & 1.7 \\
\hline$H f^{*}$ & & 2.43 & 4.0 & 5.7 & & & & \\
\hline $\mathrm{Ta}$ & 0.483 & 0.47 & 4.0 & -3.4 & 0.5 & 1.1 & 0.28 & 1.2 \\
\hline $\mathrm{Pb}$ & 7.81 & 7.63 & 9.2 & -2.3 & 0.6 & 25.2 & 0.23 & 4.4 \\
\hline Th & 2.21 & 2.28 & 3.2 & 3.2 & 0.3 & 3.6 & 0.23 & 1.7 \\
\hline $\mathrm{Th}^{*}$ & & 2.24 & 4.6 & 1.32 & & & & \\
\hline U & 0.497 & 0.509 & 5.4 & 2.4 & 0.316 & 3.4 & 0.101 & 1.6 \\
\hline$U^{*}$ & & 0.502 & 2.7 & 0.92 & & & & \\
\hline
\end{tabular}

Notes: Recommended values (RV) of W-2 standard are from Eggins et al. (1997). Abundances of Zr, Hr, Th, and U of W-2 following alkali fusion are also shown. Relative deviation (RD) values calculated between RV, values from this study, and relative standard deviation (RSD) values, an estimate of precision. For all reference samples $n=5$ except where denoted by ${ }^{*}$, where $n=8$. 
Table T4. Trace element abundances. (See table notes.) (Continued on next four pages.)

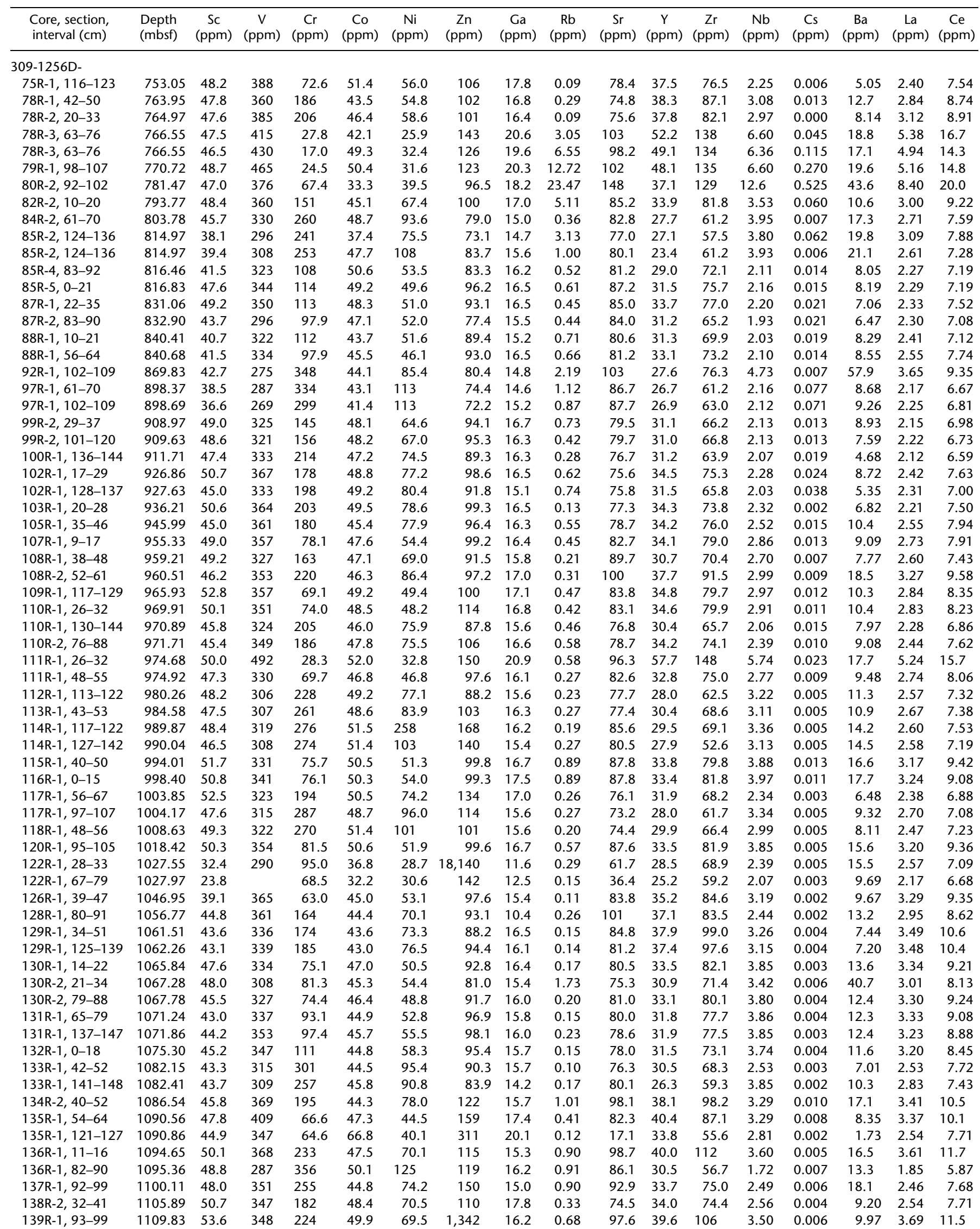


Table T4 (continued). (Continued on next page.)

\begin{tabular}{|c|c|c|c|c|c|c|c|c|c|c|c|c|c|c|c|c|c|}
\hline $\begin{array}{l}\text { Core, section, } \\
\text { interval }(\mathrm{cm})\end{array}$ & $\begin{array}{l}\text { Depth } \\
\text { (mbsf) }\end{array}$ & $\begin{array}{c}\mathrm{Pr} \\
(\mathrm{ppm})\end{array}$ & $\begin{array}{c}\mathrm{Nd} \\
(\mathrm{ppm})\end{array}$ & $\begin{array}{c}\mathrm{Sm} \\
(\mathrm{ppm})\end{array}$ & $\begin{array}{c}\mathrm{Eu} \\
(\mathrm{ppm})\end{array}$ & $\begin{array}{c}\text { Gd } \\
(\mathrm{ppm})\end{array}$ & $\begin{array}{c}\text { Tb } \\
(\mathrm{ppm})\end{array}$ & $\begin{array}{c}\text { Dy } \\
\text { (ppm) }\end{array}$ & $\begin{array}{c}\text { Ho } \\
\text { (ppm) }\end{array}$ & $\begin{array}{c}\text { Er } \\
(\mathrm{ppm})\end{array}$ & $\begin{array}{c}\mathrm{Yb} \\
(\mathrm{ppm})\end{array}$ & $\begin{array}{c}\text { Lu } \\
(\mathrm{ppm})\end{array}$ & $\begin{array}{c}\mathrm{Hf} \\
(\mathrm{ppm})\end{array}$ & $\begin{array}{c}\text { Ta } \\
(\mathrm{ppm})\end{array}$ & $\begin{array}{c}\mathrm{Pb} \\
(\mathrm{ppm})\end{array}$ & $\begin{array}{l}\text { Th } \\
(\mathrm{ppm})\end{array}$ & $\underset{(\mathrm{ppm})}{U}$ \\
\hline \multicolumn{18}{|l|}{ 309-1256D- } \\
\hline 75R-1, 1 & 3.05 & .44 & 7.90 & 3.01 & 1.13 & 4.40 & 0.86 & 5.58 & 1.30 & 3.82 & 3.76 & 0.58 & 2.10 & 0.13 & 0.15 & 0.14 & 0.062 \\
\hline & & & 8.54 & & & & & & & & & & & & & & \\
\hline $78 \mathrm{R}-2,20-33$ & & & 8.80 & & & & & & & & & & & & & & \\
\hline $78 R-3,63-76$ & 766.55 & 2.68 & 14.5 & & & & & & & & & & & & & & \\
\hline $78 R-3,6$ & & & & & & & & & & & & & & & & & \\
\hline 79R-1, 98-107 & 770.72 & 2.42 & 13.0 & .47 & & & & & & 4.82 & & & & & & & .165 \\
\hline & & & 13.5 & & & & & & & & & & & & & & \\
\hline $82 \mathrm{R}-2,10-20$ & 793.77 & 1.53 & 8.33 & 3.08 & & & & & & 3.44 & & & & & & & \\
\hline $84 \mathrm{R}-2,61-70$ & 3.78 & .20 & 6.56 & 2.28 & & & & & & 2.90 & & & & & & & \\
\hline $85 R-2,124-136$ & & .28 & 6.77 & 2.33 & & & & & & 2.76 & & & & & & & \\
\hline & & .21 & 6.22 & 2.25 & & & & & & & & & & & & & \\
\hline & & & & & & & & & & & & & & & & & \\
\hline & & & 6.90 & & & & & & & & & & & & & & \\
\hline $87 R-1,2$ & & & & & & & & & & & & & & & & & \\
\hline $87 R-2,8$ & & .23 & 7.03 & 2.50 & & & & & & & & & & & & & \\
\hline & & 2 & 7.36 & & & & & & & & & & & & & & \\
\hline & & 1.38 & 7.58 & 2.81 & & & & & & & & & & & & & \\
\hline & & & & & & & & & & & & & & & & & \\
\hline & & & & & & & & & & & & & & & & & \\
\hline & & & & & & & & & & & & & & & & & \\
\hline & & 4 & & & & & & & & & & & & & & & \\
\hline & 09.63 & 1.25 & 6.86 & 2.54 & & & & & & & & & & & & & \\
\hline & & & & & & & & & & & & & & & & & \\
\hline & & & & & & & & & & & & & & & & & \\
\hline & & & & & & & & & & & & & & & & & \\
\hline & 936.21 & 1.3 & & & & & & & & & & & & & & & \\
\hline 105R-1, 3 & 945.99 & 1.42 & 7.64 & 2.89 & & 4.2 & 0.80 & 5. & & & 3.3 & & & & & & 0.052 \\
\hline & & & & & & & & & & & & & & & & & \\
\hline & & & & & & & & & & & & & & & & & \\
\hline & & 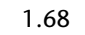 & & & & & & & & & & & & & & & \\
\hline & 965.93 & & 7.94 & & & & & & & & & & & & & & \\
\hline & 969.91 & & 7.92 & & & & & & & & & & & & & & \\
\hline & & & & & & & & & & & & & & & & & \\
\hline & & .3 & & & & & & & & & & & & & & & \\
\hline & & & & & & & & & & & & & & & & & \\
\hline 111R-1, 4 & 974.92 & 1.38 & 7.65 & 2.75 & & & & & & & 3.22 & & & & & & \\
\hline & & & & & & & & & & & & & & & & & \\
\hline & & & & & & & & & & & & & & & & & \\
\hline & & & & & & & & & & & & & & & & & \\
\hline & & & & & & & & & & & & & & & & & \\
\hline 115R-1, 40-50 & 994.01 & 1.56 & & 2.92 & & & & & & & & & & & & & \\
\hline & 998.40 & & & & & & & & & & & & & & & & \\
\hline & & & & & & & & & & & & & & & & & \\
\hline & & & & & & & & & & & & & & & & & \\
\hline & & & & & & & & & & & & & & & & & \\
\hline & & & & & & & & & & & & & & & & & 0.075 \\
\hline & & & & & & & & & & & & & & & & & \\
\hline & & & & & & & & & & & & & & & & & \\
\hline $126 \mathrm{R}-$ & & & & & & & & & & & & & & & & & \\
\hline & & & & & & & & & & & & & & & & & \\
\hline & & & & & & & & & & & & & & & & & \\
\hline & & & & & & & & & & & & & & & & & \\
\hline & & & & & & & & & & & & & & & & & \\
\hline & & & & & & & & & & & & & & & & & \\
\hline 130R-2, 79-88 & & 1.53 & & & & & & & & & & & & & & & 0.075 \\
\hline & & & & & & & & & & & & & & & & & .074 \\
\hline & & & & & & & & & & & & & & & & & \\
\hline & & & & & & & & & & & & & & & & & \\
\hline & & & & & & & & & & & & & & & & & \\
\hline 133R-1, 1 & 82.41 & 1.2 & 6.42 & 2.28 & & & & & & & 2. & & & & & & 0.072 \\
\hline & & & & & & & & & & & & & & & & & \\
\hline & & & & & & & & & & & & & & & & & \\
\hline & & & & & & & & & & & & & & & & & \\
\hline & & 1.97 & 10.7 & & & & & & & & & & & & & & 0.076 \\
\hline 136R-1, 82-90 & 1095.36 & 1.04 & & 2.39 & & & & & & & 2.97 & & & & & & 0.029 \\
\hline $137 \mathrm{P}=9290$ & & 1.37 & & 2.8 & & & & & & & & & & & & & \\
\hline & & 1.32 & & & & & & & & & & & & & & & \\
\hline 139R-1, 93-99 & 1109.83 & 1.92 & 10.5 & 3.65 & 1.25 & 5.09 & 0.95 & 5.96 & 1.38 & 3.97 & 3.75 & 0.57 & 2.73 & 0.23 & 0.33 & 0.22 & 0.070 \\
\hline
\end{tabular}


Table T4 (continued). (Continued on next page.)

\begin{tabular}{|c|c|c|c|c|c|c|c|c|c|c|c|c|c|c|c|c|c|}
\hline $\begin{array}{l}\text { Core, section, } \\
\text { interval }(\mathrm{cm})\end{array}$ & $\begin{array}{l}\text { Depth } \\
\text { (mbsf) }\end{array}$ & $\begin{array}{c}\text { Sc } \\
(\mathrm{ppm})\end{array}$ & $\begin{array}{c}\mathrm{V} \\
(\mathrm{ppm})\end{array}$ & $\begin{array}{c}\mathrm{Cr} \\
(\mathrm{ppm})\end{array}$ & $\begin{array}{c}\text { Co } \\
\text { (ppm) }\end{array}$ & $\begin{array}{c}\mathrm{Ni} \\
(\mathrm{ppm})\end{array}$ & $\begin{array}{c}\mathrm{Zn} \\
(\mathrm{ppm})\end{array}$ & $\begin{array}{c}\mathrm{Ga} \\
(\mathrm{ppm})\end{array}$ & $\begin{array}{c}\mathrm{Rb} \\
(\mathrm{ppm})\end{array}$ & $\begin{array}{c}\mathrm{Sr} \\
(\mathrm{ppm})\end{array}$ & $\begin{array}{c}\mathrm{Y} \\
(\mathrm{ppm})\end{array}$ & $\begin{array}{c}\mathrm{Zr} \\
(\mathrm{ppm})\end{array}$ & $\begin{array}{c}\mathrm{Nb} \\
(\mathrm{ppm})\end{array}$ & $\begin{array}{c}\text { Cs } \\
(\mathrm{ppm})\end{array}$ & $\begin{array}{c}\mathrm{Ba} \\
(\mathrm{ppm})\end{array}$ & $\begin{array}{c}\text { La } \\
(\mathrm{ppm})\end{array}$ & $\begin{array}{c}\mathrm{Ce} \\
(\mathrm{ppm})\end{array}$ \\
\hline 140R-1, 55-65 & 1114.72 & 55.3 & 349 & 233 & 58.8 & 72.4 & 118 & 15.4 & 0.46 & 81.5 & 39.9 & 108 & 3.53 & 0.007 & 9.46 & 3.53 & 11.3 \\
\hline $142 \mathrm{R}-1,38-48$ & 23.75 & 0.2 & 296 & & 44.9 & 120 & 134 & 14.5 & 1.48 & 89.3 & 31.0 & 81.5 & 2.86 & 0.016 & 20.7 & 3.16 & 9.68 \\
\hline $142 \mathrm{R}-2,40-55$ & 125.34 & 46.5 & 284 & 318 & 46.1 & 113 & 104 & 16.7 & 0.56 & 99.0 & 32.2 & 86.3 & 2.93 & & 7.70 & 3.37 & 10.2 \\
\hline $143 \mathrm{R}-1,72-82$ & 1128.71 & 54.1 & 330 & 194 & 49.0 & 69.5 & 80.9 & 16.1 & & 79.8 & 30.0 & 71.7 & & & 5.59 & 2.15 & 6.30 \\
\hline $143 \mathrm{R}-1,122-125$ & 1129.23 & 51.8 & 341 & 198 & 48.6 & 71.2 & 85.2 & 16.8 & 0.13 & 77.9 & 31.1 & 64.1 & 2.12 & 0.003 & 5.95 & 2.24 & 6.82 \\
\hline 143R-2, 40-49 & 1129.64 & 44.8 & 314 & 194 & 45.7 & 66.1 & 92.3 & 15.5 & 18 & 74.6 & 28.6 & 61.6 & 2.87 & 0.005 & 8.72 & 2.50 & 6.96 \\
\hline 144R-2, 39-56 & 1134.38 & 45.0 & 327 & 199 & 45.9 & 73.8 & 109 & 15.0 & 0.23 & 76.0 & 28.7 & 62.3 & 2.87 & 0.004 & 10.3 & 2.59 & 7.19 \\
\hline $145 \mathrm{R}-2,73-89$ & 1139.63 & 44.5 & 299 & 276 & 42.9 & 82.2 & 106 & 14.4 & 0.27 & 77.9 & 25.4 & 53.4 & 3.00 & & 9.44 & 2.44 & 6.85 \\
\hline $146 \mathrm{R}-1,30-54$ & 1142.50 & 43.5 & 292 & 303 & 43.6 & 83.0 & 109 & 14.3 & 0.33 & 74.3 & 24.9 & $* 58.1$ & & & 9.48 & 2.39 & 6.73 \\
\hline 146R-2, 80-88 & 1143.86 & 45.3 & 304 & 302 & 46.8 & 87.2 & 115 & 14.6 & 0.32 & 75.0 & 25.9 & $* 61.5$ & 3.10 & & 10.4 & 2.65 & 7.09 \\
\hline $147 \mathrm{R}-1,4$ & & 46.6 & 363 & 223 & 43 & 57 & 92.7 & 15.2 & & 84.8 & 32.8 & 68.7 & & & 0.8 & 2.49 & 7.47 \\
\hline $149 \mathrm{R}-1,57-63$ & 56.66 & 48.5 & 444 & 77.2 & 41.6 & 45 & 74.2 & 15.0 & & 97.1 & 46.8 & 112 & & & & 3.90 & 12.0 \\
\hline 149R-1, 138-145 & 1157.33 & 46.5 & 440 & 94.8 & 50.1 & 44.1 & 195 & 17.3 & & 88.8 & 45.2 & 110 & 8 & & 9.79 & 3.78 & 11.4 \\
\hline 151R-1, 119-125 & 1166.43 & 47.0 & 399 & 84.4 & 48.7 & 50.3 & 111 & 17.3 & 0.10 & 82.6 & 39.8 & 94.0 & 3.52 & & 9.37 & 3.38 & 9.89 \\
\hline $153 R-2,36-47$ & 1176.70 & 47.4 & 414 & 75.7 & & & 148 & 17.6 & & & & & & & 8.29 & 3.46 & 10.2 \\
\hline 153R-2, 1 & 1177.28 & 47.2 & 399 & 110 & & & 245 & 16. & & 85.9 & 38.1 & & & & & & 16 \\
\hline 154R-2, 3 & 1181.45 & 46.6 & 431 & 95.3 & 47.3 & 50.0 & 207 & 15.7 & & 94.7 & 40.6 & 95.8 & & & 18.8 & & 9.82 \\
\hline $155 \mathrm{R}-2,60-80$ & 1186.47 & 43.6 & 388 & 102 & 45.3 & & 131 & 16.2 & & 77.2 & 33.9 & *86.1 & & & 15.2 & & 59 \\
\hline 155R-3, 0-10 & 1187.12 & 43.9 & 387 & 99.3 & 46.0 & 51.1 & 118 & 16.4 & 0.11 & 69.4 & 34.8 & 102 & 3.09 & & 8.21 & 2. & 9.04 \\
\hline 156R-1, 9 & & 42.0 & 327 & 222 & 44 & & 91.7 & 14.9 & & 76.1 & 28.2 & 61.3 & & & & 2.26 & 6.68 \\
\hline 157R-1, 1 & 1195. & 43.4 & 42 & & 45 & 44 & 171 & 17.6 & & 79.1 & 42.9 & 106 & 7 & & & 65 & 11.0 \\
\hline $159 \mathrm{R}$ & 1203. & 43.2 & 40 & 8 & 46. & 46 & 144 & 17.2 & & 81.2 & 39.4 & 90.9 & & & & & 9.74 \\
\hline 159R-1, 60-70 & 1204.40 & 46.1 & 418 & 81.9 & 38.3 & 49.9 & 156 & 13.6 & 0.56 & 65.5 & 39.4 & 92.5 & 3.3 & & 11.2 & 3.07 & 9.50 \\
\hline 159R-1, 98-110 & 1204.63 & 42.3 & 371 & 163 & 45.0 & & 84.2 & 16.3 & & & & 86.7 & & & 8.19 & & 9.21 \\
\hline & & & 33 & 12 & & & 104 & & & 77.9 & 28 & & & & & & 64 \\
\hline 161 & & 39.9 & 29 & 426 & & 132 & & & & 93.2 & 25 & 50.5 & & & & & \\
\hline $162 \mathrm{R}-1,37-45$ & 1213.95 & 42.3 & 33 & 284 & & 85.8 & 88.0 & 15.7 & & & 32.3 & 80.1 & & & & 89 & 51 \\
\hline $162 \mathrm{R}-2,89-106$ & 1215.60 & 42.1 & 328 & 295 & 45.5 & 107 & 82.1 & 15.1 & 0.34 & 65.7 & 28.0 & 59.5 & 2.1 & 0.0 & 9.37 & 2.07 & 6.38 \\
\hline $163 R-2,55-63$ & 1219.91 & 45.2 & 372 & 185 & & 71.5 & 106 & 16.7 & & & & & & & & & 52 \\
\hline 164 & & 43.1 & & 278 & & & & 16. & & 84.6 & 33 & & & & & 98 & 14 \\
\hline $164 R-2,5-15$ & & 46.2 & & 260 & & & 2.0 & 16.8 & & 83.5 & 34.0 & 84.8 & & & 7.96 & 15 & 22 \\
\hline 164R-2, 101-107 & 1225.18 & 46.7 & & & 46.7 & & 77.6 & 16.9 & & 81.8 & 39.1 & 80.9 & & & 9.36 & 3.32 & 9.74 \\
\hline $164 R-3,36-44$ & 1225.86 & 45.1 & 418 & 75.5 & 45.9 & 42.3 & 67.8 & 18.1 & 0.08 & 83.6 & 42.8 & 104 & 3.67 & 0.0 & 9.03 & 3.59 & 10.9 \\
\hline 165 & & 46.6 & & & & & & & & & 38 & & & & & & .17 \\
\hline 165 & & 45.2 & 39 & 72 & 45 & & 132 & 17 & & 79.8 & 39.6 & 9 & & & 62 & 26 & 9.80 \\
\hline & & 47.1 & & & & & & 16 & & 87.1 & 40 & 94.0 & & & 9.53 & 3.12 & 9.78 \\
\hline 166R-1, 15-27 & & 44.8 & & & & & 96.9 & 16.1 & & 91.4 & 38.8 & 76.7 & & & 10.5 & 3.74 & 10.6 \\
\hline $166 \mathrm{R}-1,126-132$ & 233.74 & 42.6 & 376 & 63.5 & 41 & 43 & 138 & 15.8 & 13 & 71.9 & 38.4 & 89.0 & 3.0 & & 8.40 & 3.15 & 9.58 \\
\hline 166 & & 44.8 & 35 & & & & & & 29 & 105 & 40 & & & & & & 9.36 \\
\hline $166 \mathrm{R}-$ & & 44.3 & 39 & 66.9 & 45 & 46. & 136 & 16 & 63 & 84.5 & 41 & 95.4 & 3.4 & & 16.1 & 51 & 10.7 \\
\hline $167 \mathrm{R}-1,12-23$ & 1236.68 & 39.8 & 321 & & 40.2 & 96.4 & 75.5 & 15.1 & 34 & 67.8 & & 63.4 & 2.21 & & 10.9 & 2.13 & 6.48 \\
\hline $167 R-2,50-70$ & 1238.63 & 43.3 & 320 & 291 & 45.2 & 115 & 78.3 & 14.7 & 0.45 & 69.1 & 27.5 & 57.7 & 2.02 & 0.001 & 11.4 & 1.99 & 5.93 \\
\hline $168 \mathrm{R}-1,58-72$ & 1243.18 & 43.0 & 355 & 107 & 45.9 & & & 16.7 & & 81.1 & 37.6 & 92.2 & & & 9.95 & 3.46 & 10.3 \\
\hline & & & & & & & & & & & 36 & & & & & & 75 \\
\hline 168 & & 43.2 & & & & & & 16 & & 82 & 37 & 90.3 & & & 68 & 41 & 10.1 \\
\hline $169 \mathrm{R}-2,40-54$ & 1248.75 & 43.0 & & & & & 90.1 & 14.8 & 0.18 & 76.2 & 29.0 & 69.4 & 3.73 & & 10.7 & 2.96 & 8.02 \\
\hline $169 \mathrm{R}-3,18-37$ & 1249.88 & 41.4 & 289 & 231 & 38.8 & 76.4 & 88.6 & 12.4 & 0.65 & 93.3 & 24.3 & 53.6 & 3.01 & 0.005 & 14.3 & 2.33 & 6.40 \\
\hline $170 \mathrm{R}-2,40-48$ & 1253.50 & 42.6 & 323 & 253 & 45.3 & 78.8 & & 15.0 & 0.16 & 71.2 & 28.9 & & & & 9.73 & & 7.97 \\
\hline & & & & & & & & & & & 28 & & 7 & & 13.4 & 1.78 & 5.53 \\
\hline $170 \mathrm{R}-3,22-40$ & 254.68 & 50.5 & 265 & 365 & 48.0 & 128 & 80.6 & 15.3 & 3.15 & 95.2 & 26.5 & 49.9 & 1.64 & 0.003 & 40.7 & 1.76 & 5.42 \\
\hline \multicolumn{18}{|l|}{$312-1$} \\
\hline & & & & & & & 89.6 & & & & & 66.3 & & & 7.92 & & 7.27 \\
\hline 174 & & 46.4 & 43 & 76.9 & 47 & & 166 & 16 & & 86.4 & 37.8 & 91.4 & 3.0 & & 60.3 & 09 & 9.01 \\
\hline & & 46.3 & 39 & 128 & & & & 16 & & 82.2 & 36.5 & & & & & & 9.97 \\
\hline & & 46.5 & & & & & & 17 & & 80.1 & 41.2 & & & & & 61 & 10.8 \\
\hline & 1 & 46. & & & & & & 19 & & 86.9 & 52.7 & 141 & 5 & & 12 & 5.03 & 14 \\
\hline$R-1,51-56$ & 300.61 & 44.5 & 345 & 255 & 47. & 81 & 73.0 & 15.4 & 0.61 & 74.7 & 29.8 & & 2.00 & & 14.3 & 2.06 & 6.35 \\
\hline $182 \mathrm{R}-1,18-25$ & 1305.09 & 44.3 & 359 & 267 & 48.2 & 79.8 & 74.1 & 15.2 & 0.40 & 77.3 & 30.5 & & 1.95 & & 11.3 & 2.15 & 6.46 \\
\hline & & 47.3 & 427 & & 50.7 & & 58.5 & 17.2 & & 79.7 & 37.9 & & & & 11.1 & 2.95 & 8.84 \\
\hline & & 47.3 & & & & & & 15 & & 98.8 & & & & & & & 7.96 \\
\hline & & 46.7 & & & & & & 16.5 & & 88.7 & 35.0 & 82.4 & 2.8 & & 33.4 & 3.04 & 8.73 \\
\hline 189R-1, 0-13 & 1333.90 & 37.5 & 261 & 320 & 41.1 & 131 & 39.2 & 15.7 & 2.92 & 122 & 29.6 & 96.7 & 7.03 & 0.003 & 93.0 & 4.99 & 12.7 \\
\hline $189 \mathrm{R}-1,71-89$ & 1334.61 & 36.8 & 263 & 332 & 42.0 & 133 & 46.9 & 15.9 & 0.52 & 119 & 29.0 & 91.9 & 7.13 & 0.002 & 21.0 & 4.87 & 13.0 \\
\hline & & 44.2 & 346 & 179 & & & & 16 & & 79.6 & 33.9 & 76.7 & & & & 2.77 & 8.10 \\
\hline & & & & & & & & & & & 43 & & & & & 3.82 & 11.2 \\
\hline 205R-1, 0-4 & 882.10 & 46.4 & & 131 & & & & 18.0 & & 80.8 & 43.8 & & 3.15 & & 10.2 & 3.41 & 10.2 \\
\hline 206R-1, 8-12 & 1386.98 & 47.4 & 381 & 148 & 48.0 & 55.9 & 48.7 & 16.6 & 0.13 & 82.7 & 34.9 & & 2.87 & 0.003 & 8.54 & 2.95 & 8.52 \\
\hline $212 \mathrm{R}-1,4-7$ & 1404.14 & 45.5 & 352 & 202 & 45.0 & 59. & 77.6 & 17.3 & 0.24 & 90.5 & 35.9 & & 3.56 & 0.003 & 11.7 & 3.39 & 9.72 \\
\hline $214 \mathrm{R}-1,26-35$ & 1411.16 & 40.5 & 277 & 88.1 & 36.1 & 38.9 & 33.0 & 18.3 & 1.86 & 97.1 & 94.3 & 298 & 14.6 & 0.005 & 35.7 & 11.6 & 35.8 \\
\hline
\end{tabular}


Table T4 (continued). (Continued on next page.)

\begin{tabular}{|c|c|c|c|c|c|c|c|c|c|c|c|c|c|c|c|c|c|}
\hline $\begin{array}{l}\text { Core, section, } \\
\text { interval }(\mathrm{cm})\end{array}$ & $\begin{array}{l}\text { Depth } \\
\text { (mbsf) }\end{array}$ & $\begin{array}{c}\mathrm{Pr} \\
(\mathrm{ppm})\end{array}$ & $\begin{array}{c}\mathrm{Nd} \\
(\mathrm{ppm})\end{array}$ & $\begin{array}{c}\text { Sm } \\
(\mathrm{ppm})\end{array}$ & $\begin{array}{c}\text { Eu } \\
(\mathrm{ppm})\end{array}$ & $\begin{array}{c}\mathrm{Gd} \\
(\mathrm{ppm})\end{array}$ & $\begin{array}{c}\mathrm{Tb} \\
(\mathrm{ppm})\end{array}$ & $\begin{array}{c}\text { Dy } \\
(\mathrm{ppm})\end{array}$ & $\begin{array}{c}\text { Ho } \\
\text { (ppm) }\end{array}$ & $\begin{array}{c}\text { Er } \\
(\mathrm{ppm})\end{array}$ & $\begin{array}{c}\mathrm{Yb} \\
(\mathrm{ppm})\end{array}$ & $\begin{array}{l}\text { Lu } \\
(\mathrm{ppm})\end{array}$ & $\begin{array}{c}\mathrm{Hf} \\
(\mathrm{ppm})\end{array}$ & $\begin{array}{c}\mathrm{Ta} \\
(\mathrm{ppm})\end{array}$ & $\begin{array}{c}\mathrm{Pb} \\
(\mathrm{ppm})\end{array}$ & $\begin{array}{c}\text { Th } \\
\text { (ppm) }\end{array}$ & $\underset{(\mathrm{ppm})}{U}$ \\
\hline $140 \mathrm{R}-1,55-65$ & 1114.72 & 1.93 & 10.3 & 3.64 & 1.13 & 5.05 & 0.96 & 6.12 & 1.34 & 3.95 & 3.76 & 0.58 & 2.59 & 0.23 & 0.64 & 0.21 & 0.070 \\
\hline $142 \mathrm{R}-1,38-48$ & 23.75 & 67 & 8.69 & 3.01 & 1.05 & 4.12 & 0.76 & 4.84 & 1.05 & 3.03 & 2.87 & 0.44 & 2.00 & 0.18 & 0.11 & 0.16 & 0.048 \\
\hline $142 \mathrm{R}-2,40-55$ & 1125.34 & 1.70 & 9.14 & 3.09 & 1.09 & 4.22 & 0.77 & 4.84 & 1.10 & 3.10 & 2.96 & .46 & 2.11 & 0.18 & 0.17 & 0.17 & 0.057 \\
\hline 143R-1, 72-82 & 1128.71 & 1.12 & 6.21 & 2.40 & 0.89 & 3.56 & 0.68 & 4.47 & & 3.00 & 2.90 & & 1.63 & & & & 0.040 \\
\hline $143 \mathrm{R}-1,122-125$ & 1129.23 & 1.17 & 6.72 & 2.49 & 0.96 & 3.81 & 0.73 & 4.76 & 1.07 & 3.18 & 3.10 & 0.49 & 1.75 & 0.13 & 0.21 & 0.14 & 0.040 \\
\hline 143R-2, 40-49 & 1129.64 & 1.22 & 6.59 & 2.44 & 0.86 & 3.49 & 0.66 & 4.40 & 0.97 & 2.90 & 2.75 & 0.43 & 1.66 & 0.18 & 0.13 & 0.19 & 0.055 \\
\hline 144R-2, 39-56 & 1134.38 & 1.24 & 6.77 & 2.57 & 0.90 & 3.60 & 0.68 & 4.51 & 1.00 & 3.06 & 2.81 & 0.44 & 1.70 & 0.17 & 0.27 & 0.19 & 0.055 \\
\hline 145R-2, 73-89 & 1139.63 & 1.16 & 6.04 & 2.28 & 0.86 & 3.13 & 0.60 & 3.96 & 0.89 & 2.70 & 2.47 & 0.38 & 1.45 & 0.18 & 0.21 & 0.20 & 0.055 \\
\hline 146R-1, 30-54 & 1142.50 & 1.13 & 5.89 & 2.26 & 0.76 & 3.12 & 0.57 & 3.83 & 0.87 & 2.50 & 2.31 & 0.36 & *1.53 & 0.18 & 0.17 & ${ }^{*} 0.20$ & $* 0.06$ \\
\hline 146R-2, 80-88 & 1143.86 & 1.16 & 6.27 & 2.25 & 0.84 & 3.27 & 0.63 & 4.02 & 0.92 & 2.64 & 2.52 & 0.38 & *1.57 & 0.19 & 0.22 & ${ }^{*} 0.21$ & ${ }^{*} 0.06$ \\
\hline $147 \mathrm{R}-1,40-48$ & & 1.29 & 7.03 & 2.67 & 0.99 & 3.96 & 0.74 & 5.1 & 1.17 & 3.40 & 3.28 & 0.50 & 1.88 & 0.16 & & 0.17 & 0.051 \\
\hline 149R-1, 57-63 & 56.66 & 2.06 & 11.7 & 4.12 & 1.35 & 5.98 & 1.08 & 7.3 & 1.63 & 4.83 & 4.61 & 0.70 & 2.98 & & & & 0.083 \\
\hline 149R-1, 138-145 & 1157.33 & 1.98 & 10.7 & 3.93 & 1.37 & 5.60 & 1.01 & 6.94 & 1.56 & 4.51 & 4.34 & 0.66 & & & & & 0.077 \\
\hline 151R-1, 119-125 & 1166.43 & 1.72 & 9.27 & 3.45 & 1.20 & 4.81 & 0.91 & 6.07 & 1.37 & 4.03 & 3.93 & 0.59 & 2.41 & 0.22 & 0.37 & 0. & 0.067 \\
\hline 153R-2, 36-47 & 1176.70 & 1.79 & 9.82 & & & 5.22 & 0.97 & & & & & 0.62 & & & & 23 & \\
\hline $153 \mathrm{R}-2,1$ & 1177.28 & 1.57 & 8.5 & 3.23 & 1.16 & 4.63 & 0.91 & 5.97 & & 4.0 & $3 . \varepsilon$ & 0.5 & 2.37 & & & & \\
\hline 154R-2, 30-52 & 1181.45 & 1.79 & 9.72 & 3.43 & 1.23 & 4.98 & 0.97 & 6.40 & 1.2 & 4.20 & 4.0 & 0.63 & 2.53 & & & & \\
\hline $155 \mathrm{R}-2,60-80$ & 1186.47 & 1.59 & 8.31 & 2.99 & 1.14 & 4.44 & 0.82 & 5.38 & & 3.41 & 3.40 & 0.49 & $\star 2.21$ & & & ${ }^{*} 0.21$ & *0.07 \\
\hline 155R-3, 0-10 & 1187.12 & 1.56 & 8.49 & 3.02 & 1.07 & 4.51 & 0.80 & 5.46 & 1.21 & 3.54 & 3.49 & 0.52 & 2.01 & 0. & 0.30 & 0.18 & 0.053 \\
\hline 156R-1, 9 & & 1.1 & 6.18 & & & & & & & & & & & & & & \\
\hline 157R-1, 1 & & 1.93 & 10.5 & & 1.39 & 5.5 & 1.0 & & & 43 & & & 2.70 & & & & \\
\hline 159R-1, 3-13 & 1203. & 1.71 & 9.32 & 3.48 & 1.25 & 5.11 & 0.9 & 6.08 & 1. & 4.1 & 3. & 0. & 2. & & & & \\
\hline 159R-1, 60-70 & 1204.40 & 1.63 & 8.99 & 3.56 & 1.06 & 4.89 & 0.92 & 6.27 & 1.36 & 4.06 & 3.89 & 0.60 & 2.47 & 0.2 & 0. & 21 & 0.065 \\
\hline 159R-1, 98-110 & & 1.59 & 8.83 & & & & & & & & & & & & & & \\
\hline 160 & & 1.2 & 6.9 & & 0.92 & & & & & & & & & & & & \\
\hline 161R-1, 105-113 & & 0.91 & 5.3 & & & & & & & & & & 1. & & & & \\
\hline 162R-1, 37-45 & 1213.95 & 1.49 & 7.86 & & 1.06 & 4.08 & 0.76 & & & & & & 2.09 & & & & 062 \\
\hline 162R-2, 89-106 & 1215.60 & 1.12 & 6.22 & 2.41 & 0.89 & 3.51 & 0.65 & 4.35 & 1.00 & 2.90 & 2.81 & 0.42 & 1.62 & 0. & 0.12 & 0.13 & 0.044 \\
\hline $163 R-2,55-63$ & 1219.91 & 1.63 & 8.75 & 3.23 & & 4.61 & & & & & & & & & & & 59 \\
\hline $164 \mathrm{I}$ & & 1.5 & & & & 4.23 & & 5. & & & 0 & & 2.7 & & & & \\
\hline $164 R-2,5-15$ & 224.49 & 1.61 & 8.54 & & & 4.2 & 0.7 & & & & 3. & & 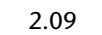 & & & & \\
\hline 164R-2, 101-107 & 1225.18 & 1.65 & 9.23 & 3.45 & 1.27 & 4.93 & & & & & & & 2.2 & & & & 064 \\
\hline 164R-3, 36-44 & 1225.86 & 1.88 & 10.3 & 3.77 & 1.33 & 5.43 & 1.00 & 6.74 & 1.52 & 4.47 & 4.29 & 0.67 & 2.68 & 0.2 & & 0.24 & 0.075 \\
\hline 165 & & 1.6 & & & & & & & & & & & 2. & & & & \\
\hline $165 \mathrm{R}-2,7$ & 12 & 1.70 & 9.36 & 3.4 & 1.24 & 5.0 & 0.9 & 6.1 & 1.3 & 4.1 & 3.94 & 0.6 & 2.39 & 0. & 3 & 0.23 & 66 \\
\hline $165 \mathrm{R}-3,1$ & & 1.67 & & & & & & & & 4 & 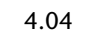 & 0.6 & 2. & 22 & & 0.22 & 0.069 \\
\hline 166R-1, 15-27 & 1232.87 & 1.81 & 9.67 & 3.54 & 1.26 & 4.94 & 0.91 & 6.09 & 1.3 & 3.96 & 3.7 & 0.5 & 2.32 & 0.27 & & 0.28 & 0.082 \\
\hline 166R-1, 126-132 & 1233.74 & 1.69 & 9.28 & 3.36 & 1.18 & 4.72 & 0.89 & 6.05 & & 3.95 & & & 2.42 & & & 0.21 & 0.062 \\
\hline $3-99$ & & 1.6 & 9.64 & & & & & & & & & & 2. & & & & \\
\hline $9-65$ & & 1.80 & 10.1 & & & & & 6.7 & & & & & 2. & & & & \\
\hline $167 \mathrm{R}-1,12-23$ & 1236.68 & & & & & & & 4.57 & & & & & 1.73 & & & & 0.047 \\
\hline $167 \mathrm{R}-2,50-70$ & 1238.63 & 1.08 & 5.78 & 2.30 & 0.85 & 3.26 & 0.62 & 4.23 & 0.96 & 2.85 & 2.67 & 0.42 & 1.52 & 0.12 & 0.10 & 0.14 & 0.042 \\
\hline $168 \mathrm{R}-1,58-72$ & 43.18 & 1.7 & 9.31 & 3.50 & 1.25 & & 0.89 & 5.93 & & 3.85 & & & & & & & 0.070 \\
\hline & & & & & & & & & & & & & & & & & \\
\hline $168 \mathrm{R}-$ & & 1.7 & & & & & & & & & & & 2. & 0. & & 22 & \\
\hline $169 \mathrm{R}-2,40-54$ & 1248.75 & 1.34 & & 2.57 & 0.91 & 3.69 & & 4.53 & & & 2.88 & 0.4 & 1.74 & & & 0.26 & 0.074 \\
\hline $169 \mathrm{R}-3,18-37$ & 1249.88 & 1.04 & 5.53 & 2.09 & 0.78 & 3.02 & 0.58 & 3.71 & 0.84 & 2.53 & 2.43 & 0.37 & 1.45 & 0. & 0. & 0.20 & 0.060 \\
\hline $170 \mathrm{R}-2,40-48$ & & & & 2.52 & 0.89 & & 0.69 & & & & 2. & & & & & & 0.068 \\
\hline & & & & & & & & & & & 2 & & 1. & & & 0 & \\
\hline $170 R-3,22-40$ & 1254.68 & 1.00 & 5.51 & 2.15 & 0.79 & 3.14 & 0.59 & 3.95 & 0.88 & 2.72 & 2.52 & 0.38 & 1.42 & 0.10 & 0.15 & 0.10 & 0.033 \\
\hline \multicolumn{18}{|l|}{$312-1$} \\
\hline & & & & & & & & & & & & & & & & & \\
\hline & & 1.5 & & & & & & & & & & 0.55 & 2.27 & 0. & 0.07 & 0.20 & 0.067 \\
\hline & & & & & & & & & & & & & & & & & \\
\hline & & 1.8 & & & & & & & & & 3.5 & 0.6 & & 3 & 0. & & \\
\hline $176 \mathrm{R}-2,4$ & & & 13.5 & 4.9 & & & & & & & 5. & 0.79 & 3.50 & 0. & 0.28 & 0.36 & 0.10 \\
\hline $181 \mathrm{R}-1,51-56$ & 1300.61 & 1.17 & 6.37 & 2.60 & 0.90 & 3.75 & 0.71 & 4.60 & 1.01 & 2.97 & 2.92 & 0.45 & & 0.11 & 0.13 & & \\
\hline $182 \mathrm{R}-1,18-25$ & 1305.09 & 1.22 & 6.63 & 2.61 & 0.95 & 3.72 & 0.70 & 4.61 & 1.03 & 2.94 & 2.92 & 0.46 & & 0.12 & 0.32 & & \\
\hline & & 1.60 & & & & & & 5.77 & & & 3.5 & & & & & & \\
\hline & & & & & & & & & & & & & & & & & 061 \\
\hline 187R-2, 21-31 & $3 \angle 5.88$ & & & & & & & 5.5 & & & 3.3 & 0.5 & 2.17 & & & 0.21 & 0.071 \\
\hline 189R-1, 0-13 & 1333.90 & 1.95 & 9.86 & 3.14 & 1.04 & 4.04 & 0.69 & 4.68 & 0.96 & 2.79 & 2.63 & 0.40 & 2.30 & 0.41 & 0.10 & 0.49 & 0.122 \\
\hline 189R-1, 71-89 & 1334.61 & 2.05 & 9.75 & 3.16 & & 4.0 & 0.71 & 4.6 & 2 & 2.78 & 2.6 & 0.40 & 2.18 & 0.43 & 0.13 & 0.46 & 0.137 \\
\hline & & 1.3 & & & & & & & & & & 0.5 & 2.03 & 0. & 0.06 & 0.17 & 0.053 \\
\hline & & & & & & & & & & & & & & & & & \\
\hline 205R-1, 0-4 & 382.10 & 1.89 & 10.4 & & & & & 6.92 & & 4.44 & 4.13 & 0.64 & & 0.21 & 0.20 & & \\
\hline 206R-1, 8-12 & 1386.98 & 1.45 & 8.21 & 3.04 & 1.08 & 4.18 & 0.81 & 5.49 & 1.18 & 3.58 & 3.39 & 0.51 & & 0.18 & 0.08 & & \\
\hline $212 \mathrm{R}-1,4-7$ & 1404.14 & 1.67 & 9.01 & 3.39 & 1.16 & 4.70 & 0.86 & 5.76 & 1.21 & 3.66 & 3.38 & 0.52 & & 0.22 & 0.28 & & \\
\hline $214 \mathrm{R}-1,26-35$ & 1411.16 & 5.89 & 30.7 & 10.1 & 3.00 & 13.2 & 2.39 & 15.7 & 3.20 & 9.75 & 8.27 & 1.18 & 7.39 & 0.87 & 0.05 & 0.79 & \\
\hline
\end{tabular}


Table T4 (continued).

\begin{tabular}{|c|c|c|c|c|c|c|c|c|c|c|c|c|c|c|c|c|c|}
\hline $\begin{array}{l}\text { Core, section, } \\
\text { interval }(\mathrm{cm})\end{array}$ & $\begin{array}{l}\text { Depth } \\
\text { (mbsf) }\end{array}$ & $\begin{array}{c}\text { Sc } \\
(\mathrm{ppm})\end{array}$ & $\begin{array}{c}\mathrm{V} \\
(\mathrm{ppm})\end{array}$ & $\begin{array}{c}\mathrm{Cr} \\
(\mathrm{ppm})\end{array}$ & $\begin{array}{c}\text { Co } \\
(\mathrm{ppm})\end{array}$ & $\begin{array}{c}\mathrm{Ni} \\
(\mathrm{ppm})\end{array}$ & $\begin{array}{c}\mathrm{Zn} \\
(\mathrm{ppm})\end{array}$ & $\begin{array}{c}\text { Ga } \\
(\mathrm{ppm})\end{array}$ & $\begin{array}{c}\mathrm{Rb} \\
(\mathrm{ppm})\end{array}$ & $\begin{array}{c}\mathrm{Sr} \\
(\mathrm{ppm})\end{array}$ & $\begin{array}{c}\mathrm{Y} \\
(\mathrm{ppm})\end{array}$ & $\begin{array}{c}\mathrm{Zr} \\
(\mathrm{ppm})\end{array}$ & $\begin{array}{c}\mathrm{Nb} \\
(\mathrm{ppm})\end{array}$ & $\begin{array}{c}\text { Cs } \\
(\mathrm{ppm})\end{array}$ & $\begin{array}{c}\mathrm{Ba} \\
(\mathrm{ppm})\end{array}$ & $\begin{array}{c}\text { La } \\
(\mathrm{ppm})\end{array}$ & $\begin{array}{c}\mathrm{Ce} \\
(\mathrm{ppm})\end{array}$ \\
\hline $214 R-1,26-35$ & 1411.16 & 72.8 & 175 & 196 & 58.8 & 53.1 & 56.9 & 25.6 & 2.37 & 149 & 52.5 & 95.3 & 4.45 & 0.006 & 42.6 & 4.80 & 14.9 \\
\hline $214 \mathrm{R}-1,52-58$ & 1411.42 & 43.5 & 186 & 49.5 & 38.5 & 29.9 & 40.8 & 18.1 & 1.37 & 86.6 & 93.2 & 336 & 15.4 & 0.007 & 21.3 & 11.7 & 36.3 \\
\hline 214R-2, 119-129 & 1413.55 & 42.1 & 276 & 325 & 37.1 & 82.3 & 34.1 & 15.9 & 0.46 & 94.1 & 27.9 & 66.7 & 2.24 & 0.002 & 10.1 & 2.52 & 7.32 \\
\hline $215 R-1,43-49$ & 1416.13 & 40.5 & 260 & 227 & 38.3 & 85.5 & 36.4 & 15.9 & 0.34 & 95.5 & 26.1 & 60.1 & 2.18 & 0.003 & 9.02 & 2.30 & 6.89 \\
\hline 216R-1, 49-58 & 1418.39 & 44.4 & 219 & 313 & 38.7 & 75.4 & 34.4 & 14.6 & 0.38 & 94.3 & 22.5 & 49.0 & 1.59 & 0.002 & 11.9 & 2.39 & 6.30 \\
\hline $217 \mathrm{R}-1,17-21$ & 1421.77 & 42.3 & 244 & 1126 & 36.7 & 147 & 25.6 & 13.1 & 0.32 & 79.6 & 16.0 & 29.9 & 1.04 & 0.000 & 8.38 & 1.61 & 4.78 \\
\hline $217 R-1,29-33$ & 1421.89 & 41.9 & 251 & 652 & 40.4 & 127 & 30.4 & 14.5 & 0.30 & 88.2 & 20.8 & 36.0 & 1.44 & 0.001 & 8.15 & 2.13 & 6.60 \\
\hline $219 \mathrm{R}-1,25-32$ & 1430.26 & 46.2 & 283 & 672 & 42.3 & 117 & 45.6 & 14.3 & 0.63 & 87.5 & 22.8 & 53.5 & 2.00 & 0.003 & 14.7 & 2.42 & 6.24 \\
\hline 220R-1, 92-100 & 1435.92 & 42.9 & 318 & 508 & 47.1 & 118 & 38.9 & 15.9 & 0.41 & 90.2 & 26.3 & 56.9 & 2.14 & 0.000 & 18.1 & 2.43 & 7.06 \\
\hline $222 \mathrm{R}-2,25-35$ & 1446.37 & 40.9 & 231 & 856 & 43.2 & 163 & 34.4 & 13.7 & 0.45 & 86.7 & 22.2 & 41.1 & 1.84 & 0.001 & 7.72 & 2.01 & 5.97 \\
\hline 223R-1, 137-144 & 1450.68 & 41.0 & 295 & 418 & 54.1 & 233 & 38.7 & 13.9 & 0.29 & 79.9 & 24.0 & 49.5 & 1.94 & 0.001 & 7.21 & 1.88 & 5.60 \\
\hline $223 R-3,12-24$ & 1452.40 & 40.4 & 203 & 549 & 65.6 & 389 & 44.6 & 13.6 & 0.32 & 88.8 & 18.7 & 35.8 & 1.27 & 0.002 & 6.55 & 1.62 & 4.68 \\
\hline 227R-1, 50-61 & 1469.00 & 45.0 & 481 & 136 & 46.3 & 68.1 & 64.3 & 17.1 & 0.17 & 80.5 & 32.0 & 70.4 & 2.73 & 0.002 & 6.69 & 2.65 & 8.13 \\
\hline 227R-1, 80-86 & 1469.30 & 47.7 & 438 & 152 & 50.2 & 69.1 & 66.7 & 17.9 & 0.12 & 76.4 & 33.9 & 72.1 & 2.67 & 0.003 & 6.71 & 2.54 & 7.96 \\
\hline 227R-1, 113-126 & 1469.63 & 44.5 & 471 & 162 & 44.1 & 75.0 & 38.4 & 17.0 & 0.38 & 85.6 & 34.5 & 86.3 & 3.18 & 0.001 & 7.44 & 3.22 & 10.1 \\
\hline $230 \mathrm{R}-1,72-83$ & 1483.72 & 49.1 & 637 & 172 & 58.2 & 74.3 & 64.5 & 18.6 & 0.22 & 85.5 & 28.4 & 53.1 & 1.92 & 0.001 & 6.46 & 2.07 & 5.91 \\
\hline 230R-2, 49-61 & 1484.99 & 45.7 & 262 & 419 & 42.7 & 88.9 & 47.2 & 15.1 & 0.27 & 89.2 & 27.0 & 45.4 & 1.52 & 0.003 & 7.86 & 1.69 & 5.38 \\
\hline 231R-1, 90-100 & 1488.80 & 46.6 & 281 & 472 & 42.6 & 98.6 & 46.2 & 15.2 & 0.18 & 87.9 & 28.9 & 52.1 & 1.79 & 0.001 & 7.12 & 1.91 & 5.96 \\
\hline 231R-3, 80-98 & 1491.36 & 46.5 & 359 & 588 & 47.1 & 109 & 50.9 & 15.4 & 0.24 & 91.7 & 24.9 & 47.2 & 1.40 & 0.002 & 7.38 & 1.51 & 4.57 \\
\hline 232R-1, 66-81 & 1493.56 & 45.4 & 329 & 487 & 48.0 & 115 & 52.0 & 15.8 & 0.31 & 92.3 & 27.9 & 45.4 & 1.57 & 0.003 & 8.94 & 1.71 & 5.51 \\
\hline $232 \mathrm{R}-2,35-50$ & 1494.33 & 45.5 & 315 & 450 & 44.6 & 97.5 & 44.6 & 15.5 & 0.15 & 89.6 & 31.0 & 65.5 & 2.25 & 0.002 & 7.39 & 2.23 & 7.23 \\
\hline 233R-1, 14-19 & 1497.64 & 45.9 & 335 & 254 & 44.6 & 80.7 & 46.6 & 15.2 & 0.16 & 74.0 & 23.9 & 43.8 & 1.34 & 0.004 & 5.24 & 1.57 & 4.50 \\
\hline 234R-1, 26-29 & 1502.76 & 45.8 & 467 & 47 & 49.8 & 42.2 & 88.4 & 18.7 & 0.30 & 79.7 & 56.6 & 149.1 & 5.22 & 0.002 & 8.08 & 4.91 & 14.6 \\
\hline
\end{tabular}

Notes: Most elements analyzed by ICP-MS using acid digestion. $\mathrm{Zr}, \mathrm{Hf}$, Th, and $\mathrm{U}$ abundances of three samples from Expedition 309 (*) and all samples from Expedition 312 were analyzed using the alkali fusion method.

\begin{tabular}{|c|c|c|c|c|c|c|c|c|c|c|c|c|c|c|c|c|c|}
\hline $\begin{array}{l}\text { Core, section, } \\
\text { interval }(\mathrm{cm})\end{array}$ & $\begin{array}{l}\text { Depth } \\
\text { (mbsf) }\end{array}$ & $\begin{array}{c}\mathrm{Pr} \\
(\mathrm{ppm})\end{array}$ & $\begin{array}{c}\mathrm{Nd} \\
(\mathrm{ppm})\end{array}$ & $\begin{array}{c}\text { Sm } \\
(\mathrm{ppm})\end{array}$ & $\begin{array}{c}\mathrm{Eu} \\
(\mathrm{ppm})\end{array}$ & $\begin{array}{c}\text { Gd } \\
(\mathrm{ppm})\end{array}$ & $\begin{array}{c}\text { Tb } \\
\text { (ppm) }\end{array}$ & $\begin{array}{c}\text { Dy } \\
(\mathrm{ppm})\end{array}$ & $\begin{array}{l}\text { Ho } \\
(\mathrm{ppm})\end{array}$ & $\begin{array}{c}E r \\
(p p m)\end{array}$ & $\begin{array}{c}\mathrm{Yb} \\
(\mathrm{ppm})\end{array}$ & $\begin{array}{c}\mathrm{Lu} \\
(\mathrm{ppm})\end{array}$ & $\begin{array}{c}\mathrm{Hf} \\
(\mathrm{ppm})\end{array}$ & $\begin{array}{c}\mathrm{Ta} \\
(\mathrm{ppm})\end{array}$ & $\begin{array}{c}\mathrm{Pb} \\
(\mathrm{ppm})\end{array}$ & $\begin{array}{c}\text { Th } \\
(\mathrm{ppm})\end{array}$ & $\begin{array}{c}U \\
(p p m)\end{array}$ \\
\hline $214 R-1,26-35$ & 1411.16 & 2.53 & 14.2 & 5.09 & 1.83 & 6.90 & 1.32 & 8.54 & 1.81 & 5.52 & 5.05 & 0.74 & 2.53 & 0.34 & 0.16 & 0.24 & 0.084 \\
\hline $214 \mathrm{R}-1,52-58$ & 1411.42 & 5.84 & 29.9 & 9.91 & 3.17 & 12.8 & 2.33 & 15.1 & 3.19 & 9.40 & 8.37 & 1.17 & 7.97 & 0.95 & 0.04 & 0.83 & 0.298 \\
\hline 214R-2, 119-129 & 1413.55 & 1.22 & 6.83 & 2.66 & 1.03 & 3.65 & 0.67 & 4.57 & 0.97 & 2.87 & 2.61 & 0.39 & 1.68 & 0.14 & 0.12 & 0.14 & 0.047 \\
\hline 215R-1, 43-49 & 1416.13 & 1.16 & 6.47 & 2.26 & 1.07 & 3.34 & 0.62 & 3.93 & 0.89 & 2.63 & 2.49 & 0.36 & 1.55 & 0.14 & 0.10 & 0.13 & 0.047 \\
\hline $216 R-1,49-58$ & 1418.39 & 1.12 & 5.76 & 2.03 & 0.93 & 2.79 & 0.54 & 3.49 & 0.77 & 2.26 & 2.15 & 0.31 & 1.21 & 0.10 & 0.10 & 0.11 & 0.042 \\
\hline $217 R-1,17-21$ & 1421.77 & 0.80 & 4.11 & 1.54 & 0.73 & 2.12 & 0.40 & 2.75 & 0.60 & 1.69 & 1.55 & 0.23 & 0.85 & 0.06 & 0.10 & 0.07 & 0.027 \\
\hline 217R-1, 29-33 & 1421.89 & 1.20 & 5.92 & 2.09 & 0.88 & 2.77 & 0.53 & 3.41 & 0.74 & 2.15 & 2.01 & 0.30 & 1.02 & 0.09 & 0.10 & 0.10 & 0.034 \\
\hline 219R-1, 25-32 & 1430.26 & 1.02 & 5.61 & 2.02 & 0.82 & 2.95 & 0.54 & 3.61 & 0.81 & 2.38 & 2.13 & 0.33 & 1.37 & 0.11 & 0.13 & 0.12 & 0.036 \\
\hline 220R-1, 92-100 & 1435.92 & 1.17 & 6.31 & 2.59 & 0.94 & 3.42 & 0.65 & 4.18 & 0.93 & 2.66 & 2.53 & 0.40 & .53 & 0.14 & 0.14 & 0.14 & 0.047 \\
\hline $222 \mathrm{R}-2,25-35$ & 1446.37 & 1.00 & 5.77 & 2.03 & 0.77 & 2.84 & 0.56 & 3.64 & 0.77 & 2.27 & 2.10 & 0.33 & 1.09 & 0.12 & 0.07 & 0.10 & 0.029 \\
\hline 223R-1, 137-144 & 1450.68 & 1.05 & 5.68 & 2.14 & 0.75 & 2.95 & 0.58 & 3.81 & 0.84 & 2.35 & 2.24 & 0.35 & 1.29 & 0.12 & 0.16 & 0.13 & 0.039 \\
\hline $223 R-3,12-24$ & 1452.40 & 0.85 & 4.33 & 1.71 & 0.69 & 2.32 & 0.44 & 2.94 & 0.65 & 1.86 & 1.75 & 0.27 & 0.98 & 0.08 & 0.10 & 0.09 & 0.026 \\
\hline 227R-1, 50-61 & 1469.00 & 1.42 & 7.95 & 2.89 & 1.05 & 3.92 & 0.78 & 5.12 & 1.12 & 3.22 & 3.13 & 0.48 & 1.90 & 0.18 & 0.04 & 0.20 & 0.052 \\
\hline $227 \mathrm{R}-1,80-86$ & 1469.30 & 1.38 & 7.73 & 2.97 & 1.07 & 4.19 & 0.79 & 5.34 & 1.16 & 3.34 & 3.25 & 0.51 & 1.93 & 0.17 & 0.05 & 0.16 & 0.064 \\
\hline 227R-1, 113-126 & 1469.63 & 1.66 & 8.78 & 3.10 & 1.05 & 4.24 & 0.80 & 5.35 & 1.21 & 3.51 & 3.28 & 0.51 & 2.11 & 0.19 & 0.08 & 0.22 & 0.065 \\
\hline 230R-1, 72-83 & 1483.72 & 1.04 & 5.98 & 2.39 & 0.95 & 3.40 & 0.65 & 4.37 & 1.00 & 2.64 & 2.73 & 0.42 & 1.40 & 0.11 & 0.02 & 0.11 & 0.035 \\
\hline 230R-2, 49-61 & 1484.99 & 0.96 & 5.83 & 2.24 & 0.94 & 3.27 & 0.64 & 4.25 & 0.95 & 2.74 & 2.58 & 0.39 & 1.28 & 0.09 & 0.10 & 0.10 & 0.028 \\
\hline 231R-1, 90-100 & 1488.80 & 1.15 & 6.59 & 2.52 & 0.99 & 3.52 & 0.70 & 4.56 & 1.02 & 2.97 & 2.69 & 0.42 & 1.48 & 0.12 & 0.08 & 0.13 & 0.037 \\
\hline 231R-3, 80-98 & 1491.36 & 0.85 & 4.80 & 1.98 & 0.90 & 3.05 & 0.60 & 3.92 & 0.86 & 2.51 & 2.43 & 0.36 & 1.31 & 0.09 & 0.08 & 0.10 & 0.034 \\
\hline $232 \mathrm{R}-1,66-81$ & 1493.56 & 1.01 & 5.88 & 2.27 & 1.00 & 3.37 & 0.66 & 4.32 & 0.97 & 2.80 & 2.63 & 0.41 & 1.31 & 0.10 & 0.11 & 0.10 & 0.032 \\
\hline $232 R-2,35-50$ & 1494.33 & 1.25 & 7.37 & 2.77 & 1.03 & 3.85 & 0.73 & 4.91 & 1.09 & 3.12 & 2.94 & 0.44 & 1.80 & 0.13 & 0.09 & 0.16 & 0.049 \\
\hline 233R-1, 14-19 & 1497.64 & 0.88 & 4.96 & 2.07 & 0.79 & 2.90 & 0.54 & 3.61 & 0.82 & 2.42 & 2.30 & 0.35 & 1.23 & 0.09 & 0.04 & 0.07 & 0.029 \\
\hline 234R-1, 26-29 & 1502.76 & 2.48 & 13.9 & 5.04 & 1.61 & 7.09 & 1.35 & 8.77 & 1.97 & 5.78 & 5.67 & 0.85 & 3.91 & 0.31 & 0.08 & 0.36 & 0.116 \\
\hline
\end{tabular}


Table T5. Comparisons of trace element abundances. (See table note.)

\begin{tabular}{|c|c|c|c|c|}
\hline & \multicolumn{2}{|c|}{ BAS-206 } & \multicolumn{2}{|c|}{ BAS-312 } \\
\hline & $\begin{array}{l}\text { Expedition } \\
312 \text { (avg.) }\end{array}$ & $\begin{array}{l}\text { This study } \\
\text { (avg.) }\end{array}$ & $\begin{array}{l}\text { Expedition } \\
312 \text { (avg.) }\end{array}$ & $\begin{array}{l}\text { This study } \\
\text { (avg.) }\end{array}$ \\
\hline \multicolumn{5}{|c|}{ Element oxide (wt\%): } \\
\hline $\mathrm{SiO}_{2}$ & 49.35 & 49.24 & 49.86 & 49.5 \\
\hline $\mathrm{TiO}_{2}$ & 2.09 & 2.10 & 1.33 & 1.336 \\
\hline $\mathrm{Al}_{2} \mathrm{O}_{3}$ & 13.87 & 13.86 & 15.41 & 15.445 \\
\hline $\mathrm{Fe}_{2} \mathrm{O}_{3}$ & 14.58 & 14.73 & 10.77 & 10.877 \\
\hline $\mathrm{MnO}$ & 0.26 & 0.26 & 0.18 & 0.184 \\
\hline $\mathrm{MgO}$ & 6.73 & 6.71 & 7.79 & 7.752 \\
\hline $\mathrm{CaO}$ & 9.92 & 9.79 & 11.89 & 11.819 \\
\hline $\mathrm{Na}_{2} \mathrm{O}$ & 2.83 & 2.71 & 2.44 & 2.351 \\
\hline $\mathrm{K}_{2} \mathrm{O}$ & 0.18 & 0.20 & 0.05 & 0.077 \\
\hline $\mathrm{P}_{2} \mathrm{O}_{5}$ & 0.19 & 0.17 & 0.09 & 0.11 \\
\hline Total: & 100.00 & 99.76 & 99.83 & 99.45 \\
\hline \multicolumn{5}{|c|}{ Element (ppm): } \\
\hline Sc & 42.59 & 51.7 & 39.99 & 45.8 \\
\hline V & 421.96 & 465.1 & 295.38 & 321.3 \\
\hline $\mathrm{Cr}$ & 73.28 & 84.6 & 259.03 & 252.2 \\
\hline Co & 50.78 & 55.1 & 44.27 & 46.7 \\
\hline $\mathrm{Ni}$ & 45.50 & 50.7 & 88.12 & 88.9 \\
\hline $\mathrm{Zn}$ & 99.29 & 133.0 & 82.75 & 89.3 \\
\hline $\mathrm{Sr}$ & 99.16 & 101.9 & 98.85 & 101.1 \\
\hline $\mathrm{Y}$ & 34.14 & 50.2 & 24.65 & 30.8 \\
\hline $\mathrm{Zr}$ & 111.19 & 128.5 & 71.43 & 79.2 \\
\hline $\mathrm{Ba}$ & 42.75 & 42.2 & 9.54 & 12.2 \\
\hline
\end{tabular}

Note: Average (avg.) values from Expedition 312 from Teagle et al. (2006). 